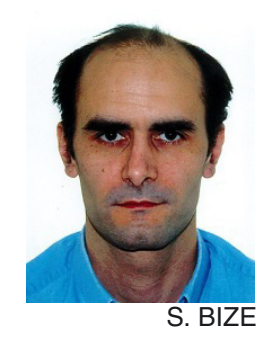

\title{
Horloge à réseau optique à atomes de mercure
}

\section{Mercury optical lattice clock}

\author{
Sébastien BIZE, Radu CHICIREANU, Samuel DAWKINS, Luigi DE SARLO, Maxime FAVIER, \\ Jocelyne GUENA, Yann LECOQ, Cipriana MANDACHE, Daniel MAGALHÃES, John MCFERRAN, \\ Sinda MEJRI, Michael PETERSEN, Rinat TYUMENEV et Lin YI
}

LNE-SYRTE, Observatoire de Paris, PSL Research University, CNRS, Sorbonne Universités, UPMC Univ. Paris 06, 61 avenue de l'Observatoire, 75014 Paris, France, sebastien.bize@obspm.fr.

\section{Résumé}

Nous décrivons le développement d'une horloge à réseau optique à atomes de mercure depuis le stade initial jusqu'aux mesures absolues qui ont permis l'inclusion de la transition horloge de ${ }^{199} \mathrm{Hg}$ dans la liste des valeurs recommandées des fréquences étalons du CIPM.

MOTS CLÉS : MÉTROLOGIE DU TEMPS ET DES FRÉQUENCES, ATOMES FROIDS, HORLOGES À RESEAU OPTIQUE, LASERS ULTRASTABLES, ATOME DE MERCURE.

\begin{abstract}
We describe the development of an optical lattice clock using mercury atoms from the initial start until absolute frequency measurements which led to including the clock transition of ${ }^{199} \mathrm{Hg}$ into the list of recommended values of standard frequencies of the CIPM.

KEYWORDS: TIME AND FREQUENCY METROLOGY, COLD ATOMS, OPTICAL LATTICE CLOCKS, ULTRASTABLE LASERS, MERCURY ATOM.
\end{abstract}

\section{Introduction}

\subsection{Contexte international : intense développement de la métrologie des fréquences optiques}

En 2000, la démonstration expérimentale d'un peigne de fréquences optiques [1,2] a déclenché une révolution. Cette méthode, fondée sur l'utilisation d'un laser femtoseconde à modes bloqués pour mesurer les fréquences optiques [3,4], a soudainement rendu accessible à une large variété de domaines de la science et de ses applications le bénéfice de plusieurs décennies d'avancées majeures dans le domaine de la spectroscopie laser et de

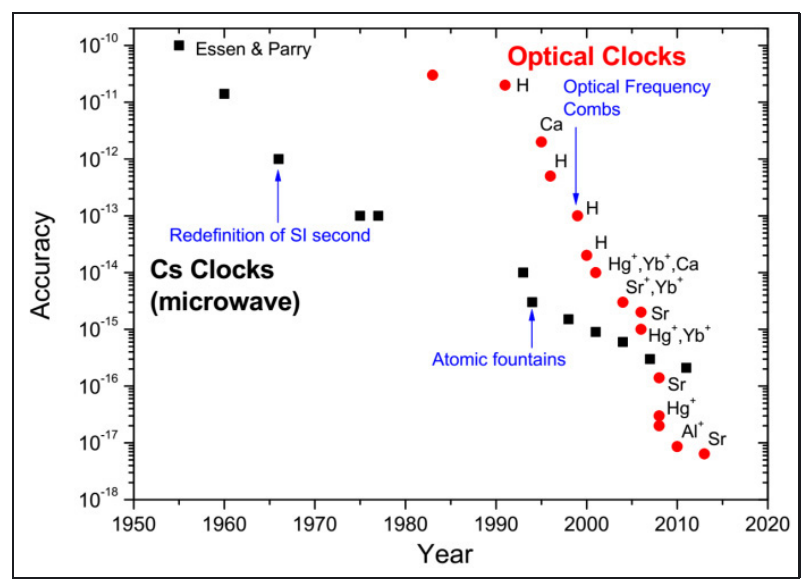

Fig. 1. - Progression de l'incertitude des étalons atomiques de fréquence.

la manipulation des atomes par laser [5-9]. Cela a notamment généré une motivation considérable au développement d'une nouvelle génération d'étalons optiques de fréquence ultraprécis, les peignes de fréquences optiques permettant l'exploitation immédiate des performances extrêmes de ces instruments. Depuis 2000, les recherches dans ce domaine ont connu une forte intensification à l'échelle mondiale, créant des conditions à la fois très stimulantes et très compétitives. La figure 1 illustre cette situation. On y montre (ronds rouges), l'amélioration de l'incertitude des étalons de fréquence optique. L'amélioration spectaculaire de plusieurs ordres de grandeurs observée depuis 2000 a conduit à ce que les étalons optiques surpassent les étalons primaires réalisés sur la base de la transition micro-onde du césium (carrés noirs sur fig. 1) de plus d'un ordre de grandeur. 


\subsection{Contribution du LNE-SYRTE au développement des horloges optiques}

Le LNE-SYRTE réalise, diffuse, utilise et développe les références primaires de fréquence et de temps pour la France. À ce titre, le LNE-SYRTE est impliqué de longue date dans la métrologie ultraprécise des fréquences optiques [10-12]. Depuis 2000, ces activités incluent le développement d'étalons de fréquence optique à atomes de strontium $(\mathrm{Sr})$. Elles ont permis, entre autres, la première excitation directe de la transition horloge $5 s^{2}{ }^{1} \mathrm{~S}_{0^{-}}$ $5 s 5 p{ }^{3} \mathrm{P}_{0}$ du ${ }^{87} \mathrm{Sr}$ [13], l'étude approfondie des effets du réseau optique établissant la possibilité d'obtenir des incertitudes extrêmes avec ce type d'étalon $[14,15]$, la première comparaison entre deux horloges à réseau optique et une série de mesures absolues de grande exactitude de la fréquence de la transition horloge du ${ }^{87} \mathrm{Sr}$ [16]. En 2005, le LNE-SYRTE a entrepris le développement d'un étalon fréquence optique à atomes de mercure qui fait l'objet du présent article.

\subsection{Principe des horloges à réseau optique}

Une horloge optique génère un signal utile situé dans le domaine optique, c'est-à-dire dont la fréquence $v_{\text {horloge }}$ est dans la gamme $10^{14} \mathrm{~Hz}$ à $10^{15} \mathrm{~Hz}$. Concrètement, c'est l'oscillation de la phase optique d'un laser continu monofréquence qui constitue la référence délivrée par ce type d'horloge. Comme dans toute horloge atomique, la fréquence de ce signal est stabilisée sur la fréquence d'une transition atomique aux propriétés adéquates. Cela est fait par un processus d'interrogation au cours duquel le laser interagit avec un ensemble d'atomes dument préparés, après quoi l'effet induit par le laser sur l'état atomique est détecté. La fréquence $10^{4}$ à $10^{5}$ fois plus élevée que celle des horloges micro-ondes, l'existence de transitions énergétiques atomiques aux propriétés intrinsèques favorables, et l'avènement de concepts et de méthodes appropriées pour les utiliser, sont à l'origine des performances extrêmes atteintes par les horloges optiques et de leur fort potentiel de progression.

Dans tout type d'horloge, il est crucial de minimiser l'influence délétère du mouvement des atomes sur le processus d'interrogation. Dans le cas des horloges à réseau optique, cela est obtenu par le piégeage des atomes dans une onde laser intense de longueur d'onde $\lambda_{\text {piège }}$. La configuration de piégeage choisie consiste en deux ondes laser gaussiennes intenses contra-propageantes formant une onde stationnaire. La figure 2 montre la distribution spatiale de l'intensité dans une telle configuration de piégeage. Selon l'axe du piège, l'interférence entre les deux ondes induit une modulation sinusoïdale de l'intensité de période $\lambda_{\text {piège }} / 2$. Dans les directions transverses, la variation d'intensité est gaussienne. L'onde laser intense exerce sur les atomes une force qu'on appelle force dipolaire. L'énergie potentielle correspondant à cette force est proportionnelle à l'intensité locale. Le mouvement des atomes est donc celui d'une particule soumise à un potentiel ayant l'allure de la distribution d'intensité. Les atomes sont attirés vers les maxima d'intensité. La varia-

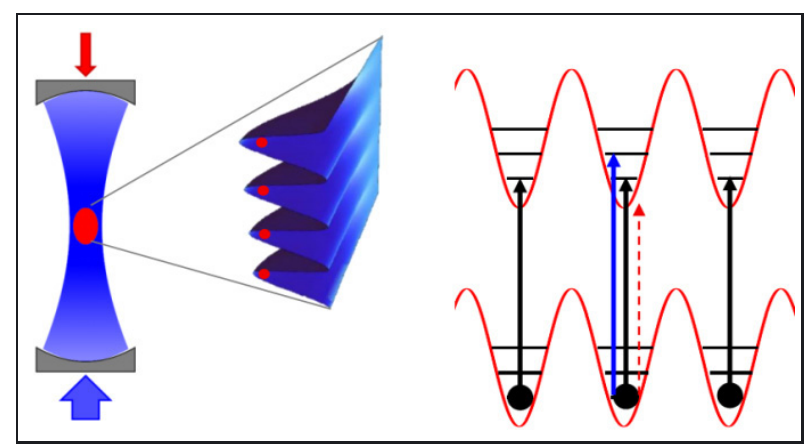

Fig. 2. - Schéma de principe d'une horloge à réseau optique.

tion rapide du potentiel sur une distance courte de $\lambda_{\text {piège }} / 2$ crée une situation de confinement fort des atomes en bas des puits de potentiel. On parle de confinement dans le réseau optique formé par l'onde intense.

Une onde laser intense perturbe les niveaux d'énergie atomiques et produit, en général, un déplacement lumineux de l'énergie de ces niveaux et de la fréquence des transitions atomiques entre ces niveaux. Pour que le piégeage décrit ci-dessus puisse être utilisé dans une horloge, il faut trouver des conditions particulières où le piégeage ne perturbe pas la fréquence de la transition horloge. Cela s'avère possible avec la transition d'intercombinaison ${ }^{1} \mathrm{~S}_{0^{-}}{ }^{3} \mathrm{P}_{0}$ des atomes de type alcalino-terreux. Dans ce cas, il existe une valeur précise de la longueur d'onde de piégeage $\lambda_{\text {piège }}$ qui annule le déplacement lumineux de la transition horloge. On parle de longueur d'onde «magique ». Dans un réseau optique à la longueur d'onde magique, on peut obtenir le confinement fort d'un nombre élevé d'atomes, sans perturber la transition horloge, ce qui crée des conditions favorables à la réalisation d'une horloge performante.

Comme indiqué dans la figure 2, l'interrogation de la transition horloge est faite au moyen d'un faisceau se propageant selon l'axe du piège. L'interrogation des atomes fortement confinés dans le réseau crée les conditions pour l'élimination très efficace des effets délétères du mouvement des atomes. Pour comprendre cet effet, il faut d'abord rappeler que le mouvement des atomes dans les puits de potentiel du réseau obéit aux lois de la mécanique quantiques. L'état de mouvement des atomes se décomposent sur un ensemble d'états vibrationnels dont les énergies possibles sont discrétisées. Pour un réseau optique à la longueur d'onde magique, le potentiel de piégeage est identique pour les deux états ${ }^{1} \mathrm{~S}_{0}$ et ${ }^{3} \mathrm{P}_{0}$ de la transition horloge. Par conséquent, la structure des niveaux vibrationnels est elle aussi la même pour les deux états, ce qui est représenté sur la figure 2. De manière générale, le processus d'interrogation sonde en même temps la structure interne et le mouvement des atomes, sans qu'il soit a priori possible de séparer l'une de l'autre. C'est par ce biais que le mouvement des atomes a un effet délétère sur l'horloge. Dans le cas d'une horloge à réseau optique et de la structure vibrationnelle discrète de la figure 2 , l'interrogation résout les diverses transitions possibles entre les structures vibrationnelles de ${ }^{1} \mathrm{~S}_{0}$ et de ${ }^{3} \mathrm{P}_{0}$. 


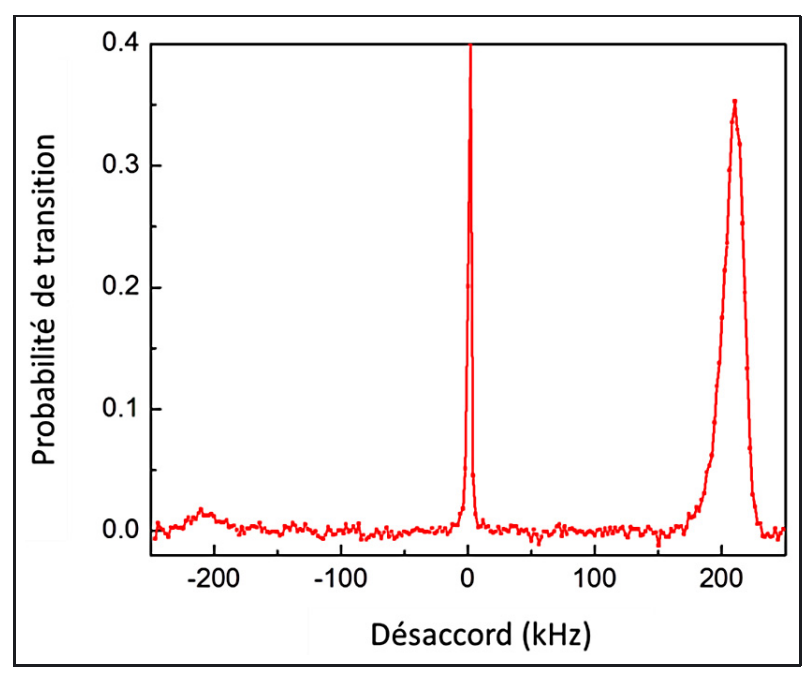

Fig. 3. - Spectroscopie de la transition horloge dans une des horloges à réseau optique à atomes de strontium du LNE-SYRTE

Le spectre de la transition se décompose ainsi en plusieurs transitions, dont certaines sont représentées sur la figure 2. Parmi ces transitions, celles qui partent et arrivent sur le même état vibrationnel ont toutes la même fréquence, qui est égale à la fréquence de la transition atomique non perturbée. Utiliser cette transition permet d'obtenir des raies expérimentales très étroites et pratiquement insensibles aux mouvements des atomes, et par conséquent, de réaliser une horloge performante.

\section{Intérêt du mercure pour une horloge optique de grande exactitude}

Cet article décrit le développement d'une horloge à réseau optique utilisant l'atome de mercure $(\mathrm{Hg})$. Dans cette section, on décrit les propriétés de l'atome de mercure qui ont conduit à ce choix.

\subsection{Niveaux d'énergie de l'atome de mercure}

La figure 4 montre les premiers niveaux d'énergie de l'atome de mercure, dont le numéro atomique est $Z=80$. La structure électronique du mercure est du type alcalinoterreux, avec deux électrons sur la couche externe. C'est une structure similaire à celle du strontium (Sr) et de l'ytterbium $(\mathrm{Yb})$, les deux principaux atomes utilisés dans les horloges à réseau optique. C'est également la structure de $\mathrm{Ca}, \mathrm{Mg}, \mathrm{Al}^{+}, \mathrm{In}^{+}$qui sont également utilisés dans des horloges optiques de grande performance. Les niveaux d'énergie atomiques de ces atomes peuvent être classés en deux catégories, selon l'appariement des spins des deux électrons externes. Dans les états singulets, les deux spins sont appariés de sorte qu'ils ont un spin total nul. C'est le cas des états ${ }^{1} \mathrm{~S}_{0}$ et ${ }^{1} \mathrm{P}_{1}$ représentés sur la partie gauche de la figure 4. Dans les états triplets, les deux spins sont appariés de sorte qu'ils ont un spin total égal à 1 . C'est le cas des états ${ }^{3} \mathrm{~S}_{1},{ }^{3} \mathrm{P}_{0},{ }^{3} \mathrm{P}_{1}$ et ${ }^{3} \mathrm{P}_{2}$ représentés à droite de la figure 4 . Les transitions entre

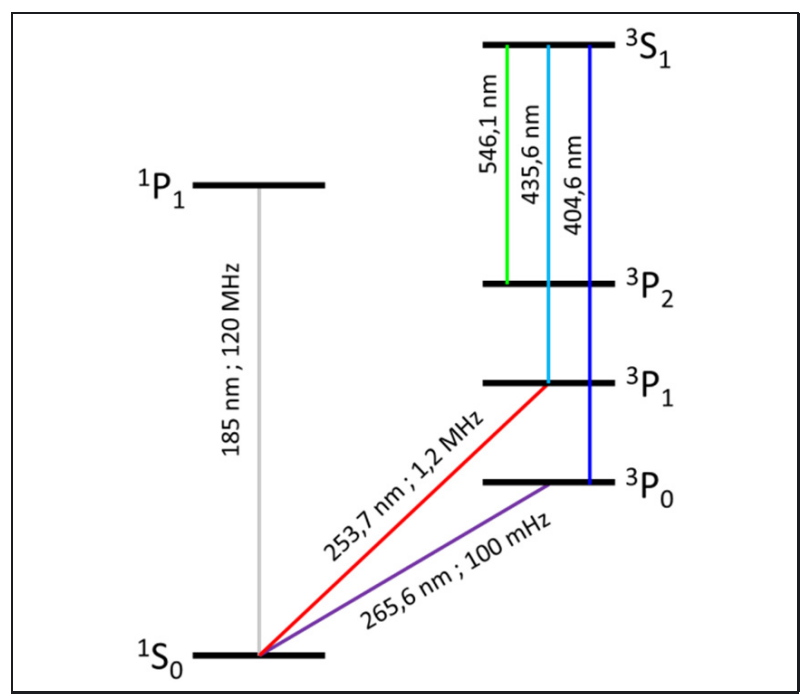

Fig. 4. - Niveaux d'énergie de l'atome de mercure.

les deux catégories d'états, singulets et triplets, sont interdites, de par la symétrie de ces états. Cela tend à créer les conditions de la métastabilité des états ${ }^{3} \mathrm{P}_{0,1,2}$ car ces états, les plus bas des triplets sur l'échelle des énergies, ne peuvent se désexciter vers l'état singulet ${ }^{1} \mathrm{~S}_{0}$. Ainsi, dans le cas du mercure, la durée de vie du niveau ${ }^{1} \mathrm{P}_{1}$, qui peut se désexciter rapidement vers le niveau ${ }^{1} \mathrm{~S}_{0}$, est de seulement $1,3 \mathrm{~ns}$, alors que la durée de vie du niveau ${ }^{3} \mathrm{P}_{1}$, déterminée par sa désexcitation vers ${ }^{1} \mathrm{~S}_{0}$ est de $125 \mathrm{~ns}$, soit presque 100 fois plus longue. On voit donc que les transitions entre un état singulet et un état triplet sont faiblement possible dans certains cas. Dans le cas de la transition de ${ }^{3} \mathrm{P}_{1}$ vers ${ }^{1} \mathrm{~S}_{0}$, le faible couplage est induit par les interactions de structure fine (interactions spin-spin, spinorbite et effets relativistes).

La figure 4 montre les longueurs d'onde et les largeurs de raies des deux transitions qu'on vient d'évoquer. On voit que la transition ${ }^{1} \mathrm{~S}_{0^{-}}{ }^{1} \mathrm{P}_{1}$ correspond à une longueur d'onde de $185 \mathrm{~nm}$ et une largeur de raie de $120 \mathrm{MHz}$. La longueur d'onde située dans le domaine ultraviolet (UV) très lointain est très difficile à synthétiser, ce qui rend cette transition inexploitable pour le refroidissement laser. La transition ${ }^{1} \mathrm{~S}_{0}-{ }^{3} \mathrm{P}_{1}$ correspond à une longueur d'onde de 253,7 $\mathrm{nm}$ et une largeur de raie de 1,2 MHz. Cette transition est utilisée pour la manipulation des atomes de mercure.

Le niveau d'énergie ${ }^{3} \mathrm{P}_{0}$ est encore plus faiblement couplé à l'état ${ }^{1} \mathrm{~S}_{0}$. La figure 4 indique, pour l'isotope fermionique ${ }^{199} \mathrm{Hg}$, la durée de vie dans ce niveau, qui vaut environ $1 \mathrm{~s}$. Ce très faible couplage est induit par l'interaction hyperfine, c'est-à-dire l'interaction des électrons avec le spin du noyau, qui est non nul pour ${ }^{199} \mathrm{Hg}$. La transition ${ }^{1} \mathrm{~S}_{0}{ }^{3}{ }^{3} \mathrm{P}_{0}$ de longueur d' onde $265,6 \mathrm{~nm}$ a ainsi une largeur de raie de $100 \mathrm{mHz}$. Cela en fait une transition horloge très étroite et donc favorable à la réalisation d'une horloge performante. Pour les isotopes bosoniques, le spin nucléaire est nul et la désexcitation vers ${ }^{1} \mathrm{~S}_{0}$ est complètement interdite. La transition peut néanmoins être utilisée. Par exemple, un champ magnétique externe 
peut être utilisé pour induire un faible couplage entre ${ }^{1} \mathrm{~S}_{0}$ et ${ }^{3} \mathrm{P}_{0}$, à la place de l'interaction hyperfine.

La figure 4 montre également trois transitions autorisées, et donc intenses, entre les niveaux énergétiques métastables ${ }^{3} \mathrm{P}_{0},{ }^{3} \mathrm{P}_{1},{ }^{3} \mathrm{P}_{2}$ et le niveau ${ }^{3} \mathrm{~S}_{1}$. Ces transitions peuvent être utilisées pour des opérations de manipulations supplémentaires des atomes.

\subsection{Propriétés pertinentes pour la réalisation d'une horloge à réseau optique}

On vient de voir que l'atome de mercure possède une transition optique très étroite, la transition ${ }^{1} \mathrm{~S}_{0}-{ }^{3} \mathrm{P}_{0}$ à $265,6 \mathrm{~nm}$, appropriée pour la réalisation d'une horloge optique. En plus de cette propriété indispensable, le mercure possède plusieurs autres propriétés particulièrement intéressantes décrites ci-après.

\subsubsection{Faible sensibilité au rayonnement thermique}

La fréquence de transition atomique est affectée par le rayonnement thermique. Le champ électromagnétique en équilibre thermodynamique avec son environnement à la température absolue $T$, appeler rayonnement du corps noir, a une densité spectrale volumique d'énergie donnée par la loi de Planck. Ce champ correspond à un champ électrique (et magnétique) fluctuant dont la valeur quadratique moyenne est donnée par :

$$
\left\langle E^{2}\right\rangle=K_{E}\left(\frac{T}{T_{0}}\right)^{4}
$$

La valeur quadratique moyenne peut s'exprimer en fonction de constantes fondamentales uniquement. Ici, on l'a exprimée en fonction d'une température de référence $T_{0}=300 \mathrm{~K} . K_{E}$ est donc la valeur quadratique du champ à $300 \mathrm{~K}$. On a $K_{E}=\left(831,9 \mathrm{~V} \cdot \mathrm{m}^{-1}\right)^{2}$. L'effet principal de ce champ fluctuant est l'effet Stark qui perturbe la fréquence des transitions atomiques de manière proportionnelle au carré du champ électrique. Dans le cas du rayonnement thermique, l'effet est principalement proportionnel à la valeur quadratique moyenne du champ. Il varie donc comme $T^{4}$. Plus précisément, la perturbation de la fréquence atomique peut s'écrire sous la forme :

$$
2 \pi \hbar \Delta v_{\text {thermique }}=\varepsilon_{0} \frac{\Delta \alpha_{a t}\left\langle E^{2}\right\rangle}{2}\left(1+\eta\left(\frac{T}{T_{0}}\right)^{2}\right) .
$$

Dans cette équation, $\Delta \alpha_{a t}$ est la différence entre les polarisabilités statiques des deux niveaux de la transition horloge. Le terme en $\eta\left(T / T_{0}\right)^{2}$ est une correction dynamique qui peut parfois excéder $10 \%$ de l'effet principal à $T_{0}$.

L'effet du rayonnement thermique doit être pris en compte dans tout type d'étalon atomique de fréquence. L'incertitude associée à cet effet vient d'une part de l'incertitude liée aux propriétés atomiques $\Delta \alpha_{a t}$ et $\eta$, et d'autre part à l'incertitude de la température absolue $T$ du rayonnement vu par les atomes. Par ailleurs, si le rayonnement vu par les atomes diffère d'un rayonnement de corps noir parfait, il faut aussi le prendre en compte. Il faut également s'assurer que les atomes ne sont pas exposés à un champ électrique statique qui produirait un déplacement Stark [17].

Le déplacement relatif de fréquence lié au rayonnement thermique à $T_{0}$ vaut $-5,3 \times 10^{-15}$ pour le strontium [18] et $-2,5 \times 10^{-15}$ pour l'ytterbium [19]. Les sensibilités correspondantes sont de $-7,1 \times 10^{-17} \mathrm{~K}^{-1}$ et $-3,3 \times 10^{-17} \mathrm{~K}^{-1}$. Par ailleurs, un champ électrique statique de $1 \mathrm{~V} \cdot \mathrm{cm}^{-1}$ induit des déplacements de fréquence de quelques $10^{-17}$. On voit donc que pour atteindre ou dépasser des exactitudes de $10^{-18}$, un effort important est nécessaire pour contrôler les effets liés à la sensibilité de la transition au rayonnement thermique et au champ électrique. Une des propriétés intéressantes du mercure est sa faible sensibilité au rayonnement thermique. Le déplacement de fréquence à $T_{0}$ est estimé à $-1,6 \times 10^{-16}$, soit 33 fois moins que pour le strontium et 16 fois moins que pour l'ytterbium, les mêmes facteurs s'appliquant pour la sensibilité au champ électrique.

\subsubsection{Absence de sensibilité tensorielle au déplacement lumineux}

Nous avons décrit le principe et l'utilité du piégeage dans un réseau optique rendu «non-perturbateur » grâce au choix précis de la longueur d'onde du piège. Étant donné l'importance de la perturbation initiale de chaque niveau d'énergie de l'horloge (qui peut être de l'ordre de $\left.10^{-10}\right)$, il est nécessaire de prendre en compte les effets de déplacement lumineux en grand détail. Ainsi, au-delà du terme scalaire principal qui est annulé à la longueur d'onde magique, la transition horloge peut-être affectée par des effets vectoriels et tensoriels et par des effets d'ordre plus élevés du couplage atome-laser. À cause de ces effets, et notamment à cause du déplacement lumineux tensoriel, la fréquence de la transition atomique est influencée par des paramètres supplémentaires comme l'état de polarisation du réseau et son orientation par rapport à la polarisation du laser d'interrogation et du champ magnétique statique définissant l'axe de quantification. Un des avantages du mercure est de posséder un isotope, ${ }^{199} \mathrm{Hg}$, dont le spin nucléaire vaut $1 / 2$. Pour cet isotope, le spin total des niveaux horloges vaut lui aussi $1 / 2$, ce qui interdit l'existence même de terme tensoriel au déplacement lumineux. La dégénérescence à seulement deux sous-états des niveaux horloges ${ }^{1} \mathrm{~S}_{0}$ et ${ }^{3} \mathrm{P}_{0}$ peut aussi permettre de simplifier et d'améliorer la sélection d'état polarisé très pur.

\subsubsection{Intérêt pour la physique fondamental et la physique atomique}

Une des applications des étalons de fréquence de grande exactitude est de tester les lois physiques fondamentales, et de contribuer ainsi à la recherche d'une théorie unifiée des interactions électrofaible, forte et gravitationnelle. Du fait du numéro atomique élevé, les niveaux 


\section{Tableau 1}

Les isotopes naturels du mercure $(Z=80)$, avec leur abondance et leur spin nucléaire. Pour les isotopes pairs, l'atome neutre est un boson et pour les isotopes impairs, c'est un fermion.

\begin{tabular}{|c|c|c|}
\hline Isotope & Abondance (\%) & Spin \\
\hline 196 & 0,15 & 0 \\
\hline 198 & 10,1 & 0 \\
\hline 199 & 17,0 & $1 / 2$ \\
\hline 200 & 23,1 & 0 \\
\hline 201 & 13,2 & $3 / 2$ \\
\hline 202 & 29,6 & 0 \\
\hline 204 & 6,85 & 0 \\
\hline
\end{tabular}

d'énergie du mercure sont en général fortement influencés par les effets relativistes. Cela confère à la transition horloge une sensibilité assez élevée à une possible variation de la constante de structure fine [20]. Cette sensibilité, combinée à la perspective d'exactitude excellente et à la diversification des systèmes atomiques qu'il apporte, font du mercure un atome particulièrement intéressant pour tester la stabilité des constantes fondamentales dans le temps, dans l'espace ou avec le potentiel de gravitation.

Le mercure possède également un grand nombre d'isotopes naturels, comme le montre le tableau 1. On a vu que l'isotope a priori favori pour la réalisation d'une horloge à réseau optique performante est l'isotope 199. Il est néanmoins possible et intéressant d'étudier le potentiel et les propriétés de chacun des autres isotopes. Le mercure n'ayant pratiquement pas été étudié en régime ultrafroid, de nombreuses propriétés restent à étudier, notamment les propriétés collisionnelles, en présence de lumière (collisions photo-associatives) ou en son absence. De telles mesures, faites pour divers isotopes, permettent d'améliorer la connaissance des propriétés atomiques et de leur modélisation théorique. Cette connaissance approfondie permettra d'envisager encore d'autres expériences exploitant les particularités du mercure, comme par exemple la mesure du moment anapolaire nucléaire.

\section{Refroidissement laser et piégeage magnéto-optique du mercure}

Au moment du démarrage du développement d'une horloge à réseau optique à atomes de mercure, cet atome n'avait jamais été refroidi par laser. Un des premiers objectifs avait donc été de démontrer et d'étudier le refroidissement laser, en commençant par concevoir et développer les méthodes et les systèmes adaptés aux spécificités du mercure. Une première spécificité vient des longueurs d'onde qui, situées dans le domaine ultraviolet, constituent un des défis de l'expérience. Une autre spécificité vient de la pression de vapeur saturante particulièrement élevée, comme on peut le voir sur la figure 5. Nous décrivons ici les systèmes qui ont été développés au LNE-SYRTE pour le refroidissement par laser des atomes de mercure.

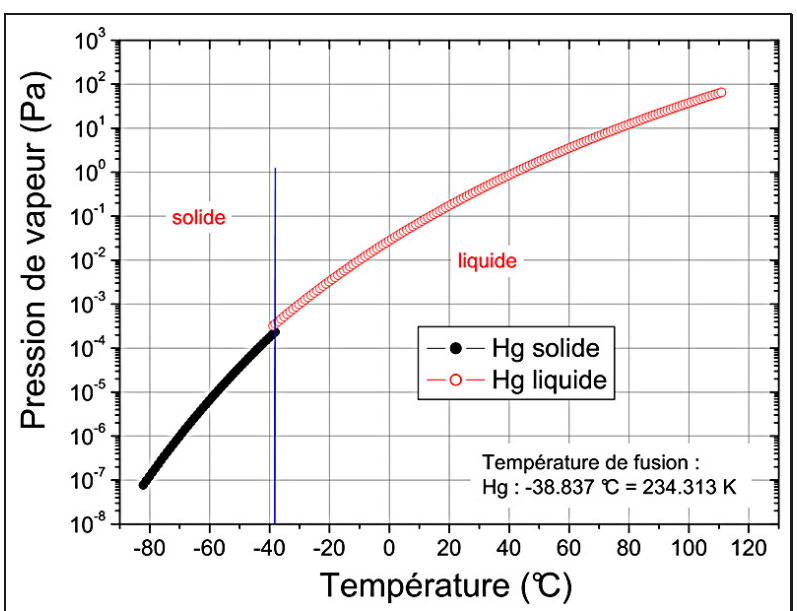

Fig. 5. - Pression de vapeur saturante du mercure. Elle est beaucoup plus élevée que celle des autres atomes utilisés dans les étalons atomiques de fréquence.

\subsection{Enceinte à ultravide}

L'enceinte à ultravide est conçue pour prendre en compte et pour exploiter les possibilités offertes par la pression de vapeur saturante élevée. Elle comporte un piège magnéto-optique, qui permet d'accumuler les atomes froids. Le piège magnéto-optique est chargé par le jet d'atomes froids généré par un piège magnéto-optique à deux dimensions, qui capture les atomes à partir d'un vapeur de mercure de pression de $10^{-5}$ à $10^{-4} \mathrm{~Pa}$. La figure 6 montre une photographie de l'enceinte à vide lors de son montage initial.

Les éléments centraux de l'enceinte à ultravide sont réalisés en alliage à base de titane. Ce matériau a l'avantage d'être à la fois excellent pour l'ultravide, non magnétique, d'avoir un faible coefficient d'expansion thermique, ce qui permet de réaliser des fenêtres collées directement verre sur métal. Les fenêtres sont en silice fondue pour l'ultraviolet et comportent, sur les deux faces, un traitement diélectrique antireflet à la longueur d'onde du laser de refroidissement. Les fenêtres sont collées avec une colle de type «époxy» compatible avec l'ultravide.

Comme le montre la figure 7 , l'enceinte est conçue pour porter et positionner précisément un ensemble de bobines portées par des supports en cuivre refroidis par une circulation d'eau. Ces bobines produisent les champs magnétiques nécessaires au PMO et au PMO-2D. Les gradients de champ magnétique appliqués au niveau des atomes sont de l'ordre de $150 \mu \mathrm{T} \cdot \mathrm{mm}^{-1}$. L'enceinte est également conçue pour porter directement certains composants optiques, comme les lames à retard $(\lambda / 4)$ et les miroirs pour le PMO et le PMO-2D.

Pour obtenir une pression de vapeur saturante de mercure de $10^{-5}$ à $10^{-4} \mathrm{~Pa}$ convenable pour un PMO-2D, il est nécessaire de refroidir le mercure à des températures allant de $-60{ }^{\circ} \mathrm{C}$ à $-40{ }^{\circ} \mathrm{C}$. La source de vapeur de mercure est représentée sur la figure 8 . Elle est constituée d'un réceptacle en cuivre contenant $1 \mathrm{~g}$ à $2 \mathrm{~g}$ de 


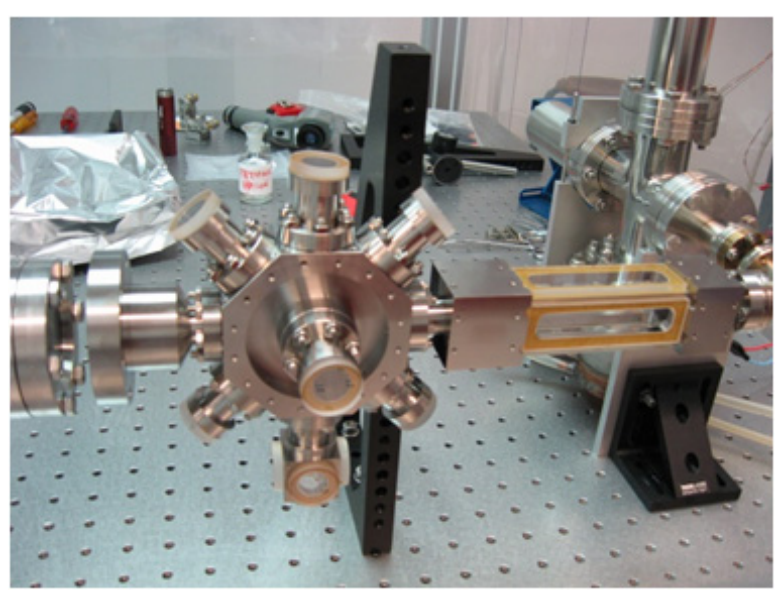

Fig. 6. - Enceinte ultravide pour le refroidissement laser et le piégeage magnéto-optique du mercure lors de son montage initial. À droite : les fenêtres rectangulaires du piège magnétooptique à deux dimensions (PMO-2D). Dans cette zone, la pression de la vapeur de mercure est de $10^{-5} \mathrm{~Pa}$ à $10^{-4} \mathrm{~Pa}$. La source de vapeur de mercure et la pompe à fixation chimique (ou pompe getter) du PMO-2D sont à l'arrière-plan de celuici. Vers le centre de la photographie : le piège magnéto-optique (PMO) avec ses fenêtres circulaires. Cette zone est pompée par une pompe ionique de $25 \mathrm{~L} / \mathrm{s}$ et par une pompe getter. La pression résiduelle y est de l'ordre de quelques $10^{-7} \mathrm{~Pa}$. Un passage de $1,5 \mathrm{~mm}$ de diamètre et de $12 \mathrm{~mm}$ de longueur, de faible conductance, permet de maintenir ce rapport de pression de 2 à 3 ordres de grandeur entre le PMO et le PMO-2D.

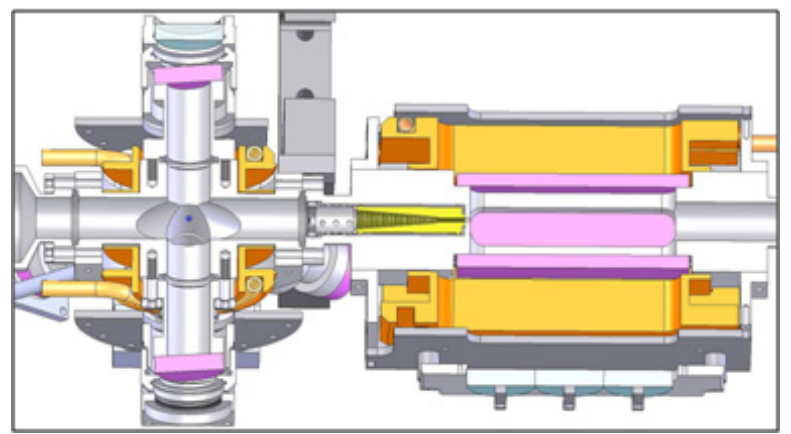

Fig. 7. - Représentation tridimensionnelle en coupe selon l'axe principal de l'enceinte à ultravide avec les bobines et les optiques attenantes.

mercure, refroidi par un module Peltier à deux étages situé dans l'enceinte à ultravide. L'ensemble est connecté au PMO-2D. En réglant le courant alimentant le module Peltier, on règle la température du réceptacle en cuivre et donc la pression de vapeur de mercure au niveau du PMO-2D.

\subsection{Source laser à $253,7 \mathbf{n m}$ pour le refroidissement}

La transition utilisée pour le refroidissement laser et le piégeage magnéto-optique du mercure est la transition ${ }^{1} \mathrm{~S}_{0^{-}}{ }^{3} \mathrm{P}_{1}$ à $253,7 \mathrm{~nm}$, dont la largeur naturelle est de $\Gamma=$ $1,2 \mathrm{MHz}$ et l'intensité de saturation de $101 \mu \mathrm{W} \cdot \mathrm{mm}^{-2}$. Pour réaliser le refroidissement par laser dans de bonne condition, il a donc fallu développer une source laser continue, monofréquence, stabilisée et néanmoins dyna-

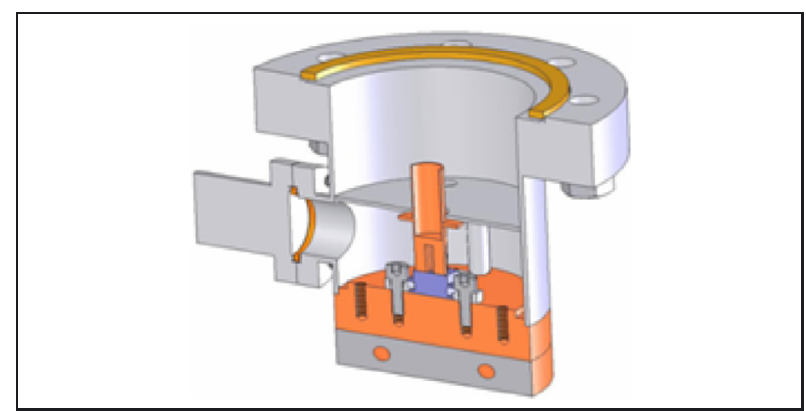

Fig. 8. - Source de vapeur de mercure pour le PMO-2D. Le tube en cuivre au centre est rempli de mercure. Il est refroidi avec un module Peltier (en violet).

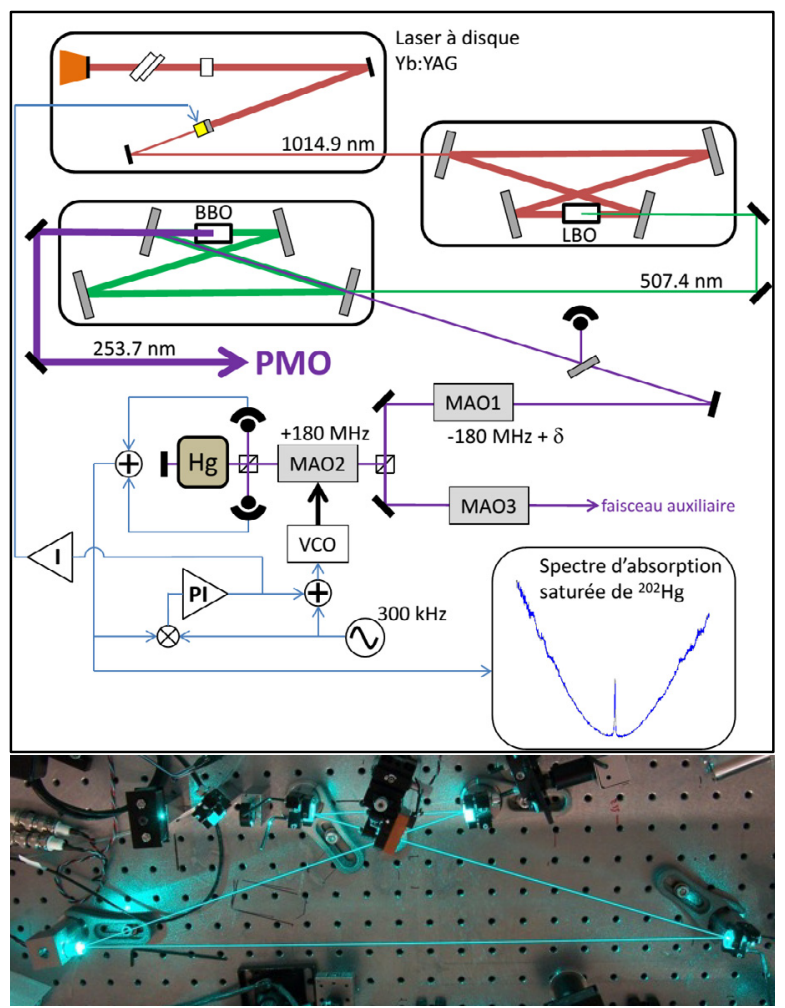

Fig. 9. - Schéma de la source laser à 253,7 nm utilisée pour le refroidissement. La photographie montre une des réalisations de la cavité de doublage produisant la lumière à $253,7 \mathrm{~nm}$, lors de tests. La diffusion Rayleigh dans l'air rend visible le faisceau à 507,4 nm à l'intérieur de la cavité où circule une puissance de plusieurs dizaines de watts.

miquement accordable, ayant une largeur de raie petite devant la largeur naturelle et d'une puissance de l'ordre de $100 \mathrm{~mW}$.

La figure 9 est un schéma de cette source. La lumière à 253,7 nm est générée par deux doublages successifs en partant de $1014,9 \mathrm{~nm}$. Ces deux doublages sont réalisés en utilisant des cavités de surtension en anneau de type «bow-tie» asservies sur la fréquence de la lumière laser incidente. Les éléments permettant ces asservissements ne sont pas représentés sur la figure 9. Le premier doublage est réalisé avec un cristal de triborate de lithium, $\mathrm{LiB}_{3} \mathrm{O}_{5}$ (LBO) taillé pour un accord de phase non-critique de type I. La température d'accord 
de phase est de $215^{\circ} \mathrm{C}$. Le second doublage est réalisé avec un cristal de bêta-borate de baryum, $\beta-\mathrm{BaB}_{2} \mathrm{O}_{4}$ (BBO) taillé pour un accord de phase angulaire de type I $\left(\theta=51,1^{\circ}, \varphi=0^{\circ}\right)$ vers $25^{\circ} \mathrm{C}$. La source laser de départ à $1014,9 \mathrm{~nm}$ est un laser à disque fin $\mathrm{Yb}$ :YAG. Du fait du vieillissement de ce laser et d'un problème de tenue à la puissance du second doublage générant le 253,7 nm, il y a eu, pendant le déroulement du projet, de grandes disparités entre les puissances lasers maximales obtenues et les puissances concrètement utilisées pour faire fonctionner l'horloge. Ainsi, les puissances à $1014,9 \mathrm{~nm}$ en sortie du laser à disque ont pu aller de $3 \mathrm{~W}$ à $8 \mathrm{~W}$, les puissances à $507,4 \mathrm{~nm}$ de $1 \mathrm{~W}$ à $3,5 \mathrm{~W}$, les puissances à $253,7 \mathrm{~nm}$ de $40 \mathrm{~mW}$ à $800 \mathrm{~mW}$. Le système de stabilisation de la fréquence de la source à $253,7 \mathrm{~nm}$ est représenté sur la figure 9. Le laser à disque comporte le disque en $\mathrm{Yb}$ :YAG brasé à un doigt en cuivre permettant une évacuation efficace de la chaleur (en haut à gauche de fig. 9). Une cavité externe est réalisée à partir de ce disque. Elle comporte un filtre de Lyot, un étalon contrôlé en température, un miroir servant à replier la cavité (pour limiter la taille du système) et un coupleur de sortie de réflectivité $99 \%$ monté sur une cale piézoélectrique permettant le contrôle fin de la fréquence. La longueur de la cavité laser est proche de $700 \mathrm{~mm}$. Comme indiqué sur figure 9, la stabilisation de la fréquence est réalisée à partir du faisceau laser issue de la réflexion partielle sur le coupleur de sortie de la seconde cavité de doublage. La fréquence de ce faisceau est décalée au moyen de deux modulateurs acousto-optique successifs (MAO1 et MAO2), après quoi il entre dans le dispositif permettant d'observer le spectre d'absorption saturée d'une vapeur de mercure. La vapeur est confinée dans une cellule en quartz de grade UV scellée et laissée à la température du laboratoire. La longueur d'interaction avec la vapeur est de $1 \mathrm{~mm}$ seulement, du fait de la pression de vapeur élevée du mercure. La figure 9 montre l'allure du spectre d'absorption saturée de la transition ${ }^{1} \mathrm{~S}_{0^{-}}{ }^{3} \mathrm{P}_{1}$ pour l'isotope ${ }^{202} \mathrm{Hg}$. La largeur $\mathrm{du}$ pic de saturation est proche de la largeur naturelle, soit 1,2 MHz. La largeur du pic Doppler (qu'on ne voit pas intégralement sur la fig. 9) est de $980 \mathrm{MHz}$. On observe des spectres similaires pour les autres isotopes. À certains endroits du spectre complet de la raie ${ }^{1} \mathrm{~S}_{0}{ }^{3} \mathrm{P}_{1}$, il y a chevauchement entre les raies Doppler des divers isotopes, sans que cela ne pose de difficulté pour l'utilisation décrite ici. Le signal d'erreur $\varepsilon$ nécessaire à l'asservissement de fréquence est généré au moyen d'une détection synchrone. Pour cela, comme indiqué sur figure 9, une modulation de la fréquence à $300 \mathrm{kHz}$ est appliquée à l'oscillateur alimentant le $\mathrm{MAO} 2$. Une première boucle d'asservissement, avec un gain proportionnel et intégral, stabilise la fréquence du faisceau sondant la cellule sur la raie atomique en agissant sur l'oscillateur du MAO2. Cet oscillateur est ainsi utilisé en filtre suiveur. Une seconde boucle d'asservissement, avec un gain intégral, corrige la fréquence du laser à disque en agissant sur la cale piézoélectrique, stabilisant ainsi en fréquence l'ensemble du dispositif.

Une série de tests non décrits dans cet article indique que la largeur de raie de la source ainsi réalisée est in- férieure à $100 \mathrm{kHz}$ à la longueur d'onde de 253,7 nm. Pour le piégeage magnéto-optique et le refroidissement par laser, il est nécessaire d'ajuster finement et de manière dynamique, le désaccord de la source laser. Pour cela, on utilise le modulateur acousto-optique MAO1. Celuici est alimenté avec une fréquence de $(180 \mathrm{MHz}-\delta)$ et est réglé pour produire un décalage de fréquence négatif, tandis que le $\mathrm{MAO} 2$ produit un décalage positif de $180 \mathrm{MHz}$. Ainsi, la fréquence alimentant le PMO se trouve décalée en fréquence de $\delta$ par rapport à la raie sur laquelle le système est asservi. Le système de contrôle de l'horloge permet de changer $\delta$ de manière dynamique et, pour que le système reste asservi lors de ces changements, les cales piézoélectriques du laser à disque et des systèmes de doublages sont actionnées de manière coordonnée avec les variations de $\delta$. Typiquement $\delta$ varie de $-6 \Gamma$, pendant une phase de capture des atomes de $1 \mathrm{~s}$ à $2 \mathrm{~s}$, à $-3 \Gamma$, pendant une phase compression et de refroidissement du PMO de $20 \mathrm{~ms}$, puis à $-1 \Gamma$, pour la phase de détection qui dure $10 \mathrm{~ms}$ et intervient après un délai variable allant de 0 à plusieurs centaines de millisecondes. Les valeurs précises des paramètres dépendent de l'isotope et du but particulier de la séquence de mesure.

\subsection{Systèmes de détection}

Les atomes de mercure sont détectés par l'intermédiaire de la lumière de fluorescence induite par la source laser de la transition à 253,7 nm. Cette longueur d'onde est accessible aux photodétecteurs en silicium, à condition que les fenêtres et composants optiques soient choisis pour transmettre le rayonnement UV. En général du quartz de grade UV est utilisé. Ainsi, on peut trouver des photodiodes ayant une efficacité quantique supérieure à $50 \%$, et des caméras CCD avec une efficacité quantique de $33 \%$ à la longueur d'onde d'intérêt. Au cours du projet, les deux possibilités ont été utilisées. Ici, on ne décrit que la détection utilisant une caméra $\mathrm{CCD}$, qui est celle utilisée pour les expériences récentes et le fonctionnement en horloge.

La figure 10a montre le schéma du système optique de la détection. L'axe optique du système de détection coïncide avec l'axe d'un des faisceaux du PMO. Comme indiqué sur la figure, la lumière de fluorescence issue des atomes d'abord imagée à l'infini avec une première lentille. Cette même lentille focalise le faisceau du PMO, ce qui permet la séparation spatiale de la lumière de fluorescence (environ $1 \mathrm{nW}$ pour $10^{6}$ atomes) et de celle du faisceau $(>10 \mathrm{~mW})$. La lumière de fluorescence des atomes est ensuite imagée sur la caméra CCD, en passant par un diaphragme réduisant le champ à la proximité du nuage atomique. La caméra CCD présente un ensemble de caractéristiques qui lui confèrent une efficacité quantique de $33 \%$ à 253,7 nm. C'est une caméra à capteur refroidi, à multiplicateur d'électrons et éclairée par l'arrière, avec une fenêtre en quartz de grade UV avec un traitement antiréflexion pour l'UV.

Le système de détection permet d'observer le PMO de manière continue, donc en présence du faisceau laser colinéaire à la détection. Toutefois, dans la plupart des 


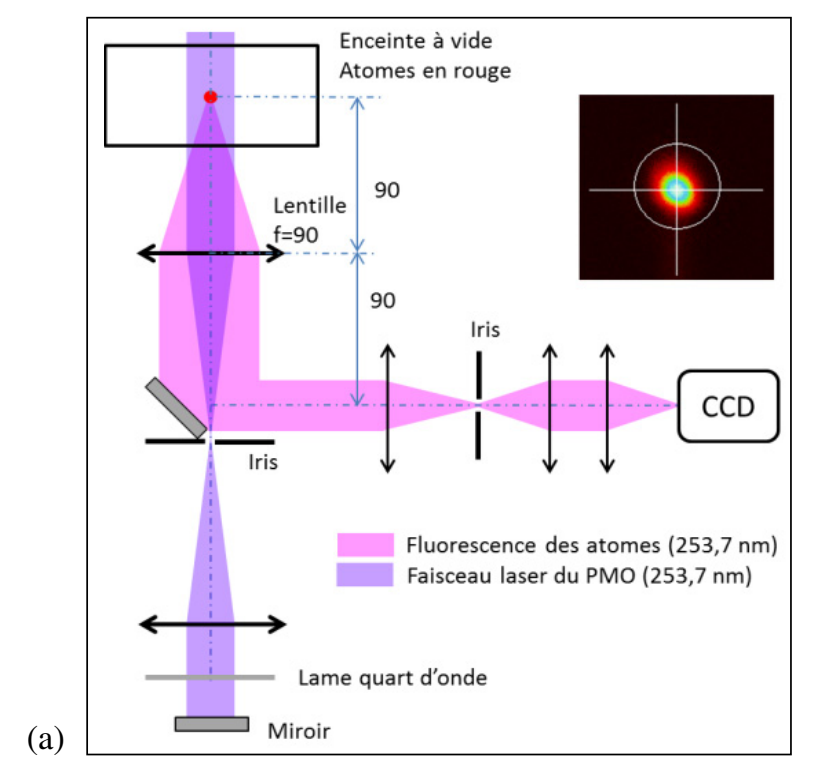

(a)

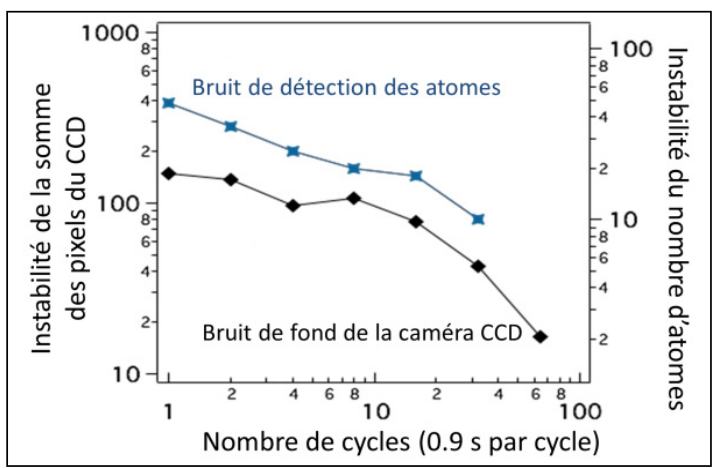

Fig. 10. - (a) Schéma de la détection utilisant une caméra CCD (les distances indiquées sont en millimètres) et image des atomes dans le PMO; (b) mesure du bruit obtenu pour la détection des atomes piégés dans le réseau optique. Le bruit à un coup équivaut à environ 40 atomes.

cas, la détection est effectuée en n'allumant que les deux faisceaux du PMO perpendiculaires à la détection. Dans ces conditions, la lumière parasite et son impact sur le bruit de détection sont minimisés. La durée d'exposition de la caméra CCD est de 5 ms. Partir des caractéristiques des éléments de la chaîne de détection, on peut traduire le signal de la caméra CCD en nombre d'atomes et exprimer le bruit de la détection en nombre d'atomes. La figure 10b présente une mesure de bruit et sa traduction en nombre d'atomes. Le bruit coup à coup du système de détection est de 40 atomes.

\subsection{Optimisation et caractérisation du refroidissement laser et du piégeage magnéto-optique}

L'ensemble des systèmes décrits ci-dessus a permis d'obtenir le refroidissement laser et le piégeage magnétooptique du mercure et de procéder à certaines optimisations en vue du transfert vers le réseau optique et de l'utilisation pour l'horloge. La figure 11a montre une courbe de chargement du PMO pour l'isotope ${ }^{202} \mathrm{Hg}$. La figure $11 \mathrm{~b}$ montre, en coordonnées logarithmiques, une

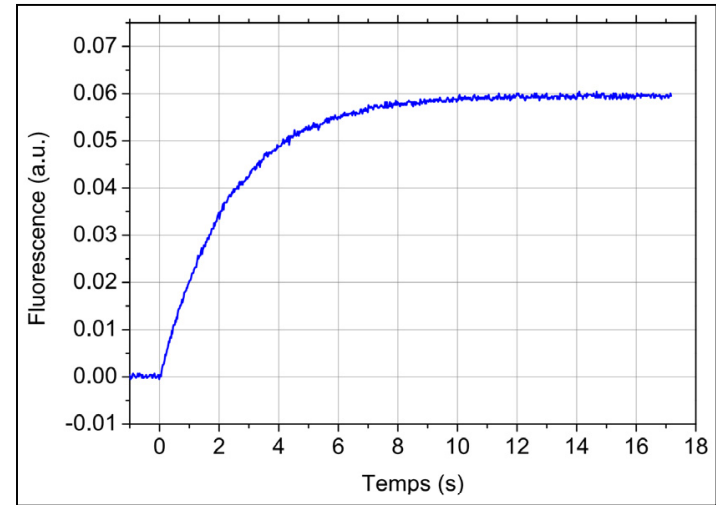

(a)

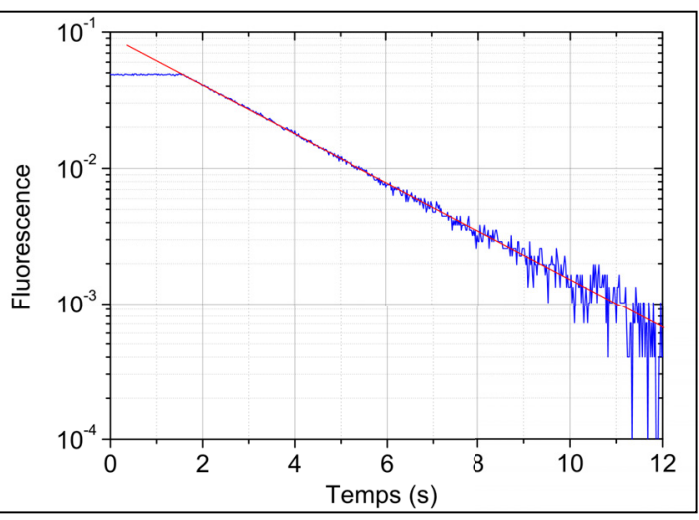

Fig. 11. - (a) Courbe de chargement du piège magnéto-optique $\mathrm{de}^{202} \mathrm{Hg}$ : sur cet exemple, la constante de temps de chargement est de 2,3 s. On estime que cette valeur est déterminée par les collisions des atomes piégés avec les atomes du gaz résiduels. (b) Nombre d'atomes (en coordonnées logarithmiques) dans le piège magnéto-optique après l'extinction du piège magnétooptique à deux dimensions.

courbe de durée de vie du PMO lorsque le piège magnétooptique à deux dimensions est coupé. Dans les conditions de cette mesure, le PMO-2D avait pour effet d'augmenter le nombre d'atomes par un facteur 5. À partir de l'analyse de ce type de courbe, nous avons constaté que la courbe de durée de vie est bien décrite par une décroissance exponentielle et la constante de temps de chargement coïncide avec la constante de durée de vie. Nous avons aussi mesuré la constante de chargement en fonction de la pression dans le PMO-2D [21]. L'ensemble de ces observations indiquent que, dans les conditions de notre expérience, la durée de vie des atomes dans le PMO est principalement limitée par les collisions avec le gaz résiduel, et qu'elle est peu influencée par les effets de densité. Nous avons observé des durées de vie allant jusqu'à $2,5 \mathrm{~s}$ pour une pression de gaz résiduel estimée à $3 \times 10^{-7} \mathrm{~Pa}$.

Le nombre d'atomes dans le PMO est un paramètre essentiel pour l'horloge et, de manière générale, pour toute expérience utilisant le PMO comme source initiale d'atomes froids. Lors le PMO a été obtenu pour la première fois, nous avons estimé le nombre d'atomes par fluorescence, mais également par absorption, méthode plutôt plus exacte, en utilisant le faisceau auxiliaire de 


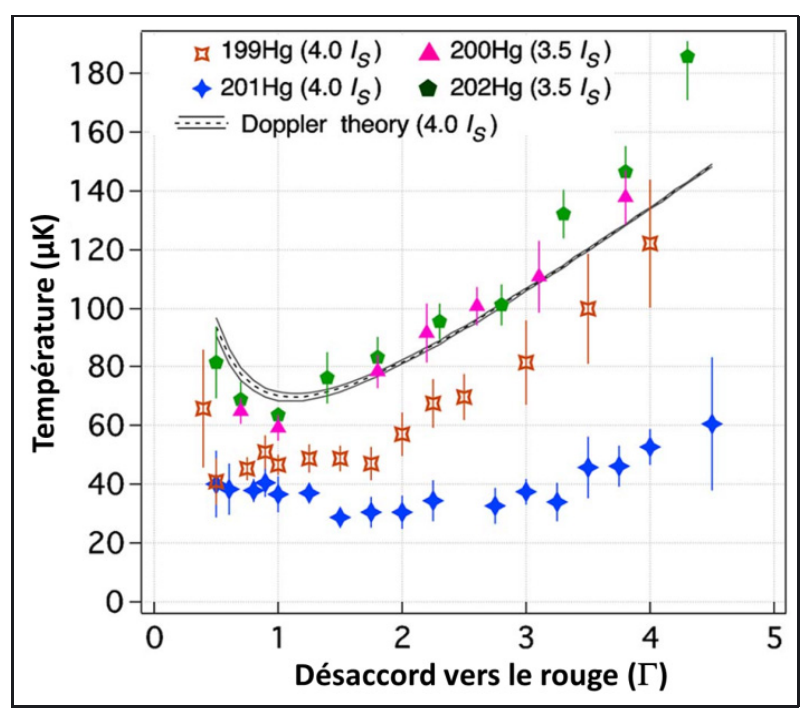

Fig. 12. - Mesures de la température du nuage atomique en fonction du désaccord pour quatre isotopes du mercure et comparaison avec une modélisation théorique du refroidissement Doppler. Pour les isotopes ${ }^{199} \mathrm{Hg}$ et ${ }^{201} \mathrm{Hg}$, on observe un refroidissement sub-Doppler.

la figure 9. Les deux mesures ont été trouvées en accord à mieux qu'un facteur deux près, ce qui est satisfaisant pour ce type de mesure. Nous avons observé des nombres d'atomes allant jusqu'à $10^{7}$ pour l'isotope ${ }^{202} \mathrm{Hg}$ le plus abondant.

Un autre paramètre déterminant pour le transfert des atomes dans le réseau optique est la température. Au cours du développement de l'expérience, la première indication concernant la température a été fournie, pour les isotopes fermioniques ${ }^{199} \mathrm{Hg}$ et ${ }^{201} \mathrm{Hg}$, par la spectroscopie de la transition horloge sur les atomes en chute libre lâchés à partir du PMO (voir plus loin). Nous avons également effectué une mesure avec le système de détection de la figure 10. La méthode consiste à lâcher les atomes à partir du PMO en arrêtant brusquement les faisceaux laser, puis à faire l'image du nuage atomique après un temps de vol balistique. En analysant des séries d'images prises pour plusieurs temps de vol, on peut déterminer à la fois la température et la taille initiale du nuage. À partir de ces mesures, nous avons étudié et optimisé le refroidissement laser pour plusieurs isotopes [22]. La figure 12 montre des mesures de la température pour quatre isotopes ${ }^{199} \mathrm{Hg},{ }^{200} \mathrm{Hg},{ }^{201} \mathrm{Hg}$ et ${ }^{202} \mathrm{Hg}$, en fonction du désaccord du laser de refroidissement. À partir de ces mesures, nous pouvons constater que pour ${ }^{200} \mathrm{Hg}$ et ${ }^{202} \mathrm{Hg}$, il y a un bon accord avec la théorie du refroidissement Doppler. Pour les isotopes ${ }^{199} \mathrm{Hg}$ et ${ }^{201} \mathrm{Hg}$, on observe un comportement différents et des températures nettement inférieures, qui sont le signe de mécanismes de refroidissement sub-Doppler. Ces observations sont conformes aux attentes. En effet, pour les isotopes ${ }^{200} \mathrm{Hg}$ et ${ }^{202} \mathrm{Hg}$, l'état fondamental ${ }^{1} \mathrm{~S}_{0}$ de la transition de refroidissement n'est pas dégénéré. Ces isotopes ne peuvent pas être le siège de mécanismes sub-Doppler. $\mathrm{Au}$ contraire, ${ }^{199} \mathrm{Hg}$ et ${ }^{201} \mathrm{Hg}$ ont un état fondamental dégénéré rendant possible les mécanismes sub-Doppler. Les mesures montrent aussi des températures plus basses pour ${ }^{201} \mathrm{Hg}$ pour lequel la dégénérescence est plus élevée (quatre sous-états au lieu de deux pour ${ }^{199} \mathrm{Hg}$ ), ce qui est conforme aux prédictions théoriques. Les températures observées pour ${ }^{199} \mathrm{Hg}$ et ${ }^{201} \mathrm{Hg}$ et notamment les températures les plus basses, proches de $30 \mu \mathrm{K}$, sont en accord avec les mesures initiales reposant sur la spectroscopie de la transition horloge.

Enfin, nous avons observé des tailles initiales du nuage atomique descendant jusqu'à $100 \mu \mathrm{m}$ (valeur quadratique moyenne de la distribution dans une direction) et des densités atomiques pouvant atteindre $1,8 \times 10^{8} \mathrm{~mm}^{-3}$, une valeur plutôt élevée et favorable pour le chargement du réseau optique.

\section{Laser ultrastable pour la transition horloge du mercure}

La transition horloge du mercure est la transition ${ }^{1} \mathrm{~S}_{0^{-}}{ }^{3} \mathrm{P}_{0}$ à $265,6 \mathrm{~nm}$. Réaliser une source laser ultrastable directement à cette longueur est difficile, principalement pour des raisons pratiques liées au manque de certains composants optiques ou optoélectroniques adéquats, comme par exemple des fibres optiques. Par conséquent, la lumière ultrastable pour l'horloge à réseau optique à atomes de mercure est générée dans le domaine infrarouge. La longueur d'onde horloge est ensuite atteinte par deux doublages successifs. Le lien entre l'horloge à réseau et le peigne de fréquences optiques est, luiaussi, fait par le biais de la lumière infrarouge. On décrit ici l'architecture et les caractéristiques de ces systèmes.

\subsection{Source laser ultrastable à $1062,5 \mathrm{~nm}$}

La source laser ultrastable à $1062,5 \mathrm{~nm}$ repose sur un laser à fibre dopée $\mathrm{Yb}$. Ce laser comporte une section source, qui est monofréquence, accordable par sa température et par une cale piézoélectrique, et une section amplificatrice qui permet d'obtenir une puissance jusqu'à $200 \mathrm{~mW}$ à la sortie d'une fibre monomode à maintien de polarisation. Nous avons déterminé que la largeur de raie de ce laser, avant toute forme de stabilisation, était de $3 \mathrm{kHz}$. Il s'agit donc d'une source laser peu bruitée et compacte, constituant un très bon point de départ pour le système laser d'interrogation d'une horloge. Ce laser à fibre est stabilisé une cavité de Fabry-Perot ultrastable, en utilisant la méthode de détection de Pound-DreverHall [23]. Plus de détails sur le système optoélectronique ont été rapportés par Dawkins et al. [24].

Les qualités du laser stabilisé pour son utilisation dans l'horloge sont principalement déterminées par la cavité ultrastable de référence. La figure 13 montre une vue en coupe de cette cavité, de ses écrans et de son enceinte à vide. Le corps de la cavité (au centre, en jaune) est en verre commercial ULE à très faible coefficient d'expansion thermique. Elle a une longueur optique de $10 \mathrm{~cm}$ et l'axe optique est disposé verticalement. Les dimensions de la cavité et la géométrie des points d'appuis, proches 


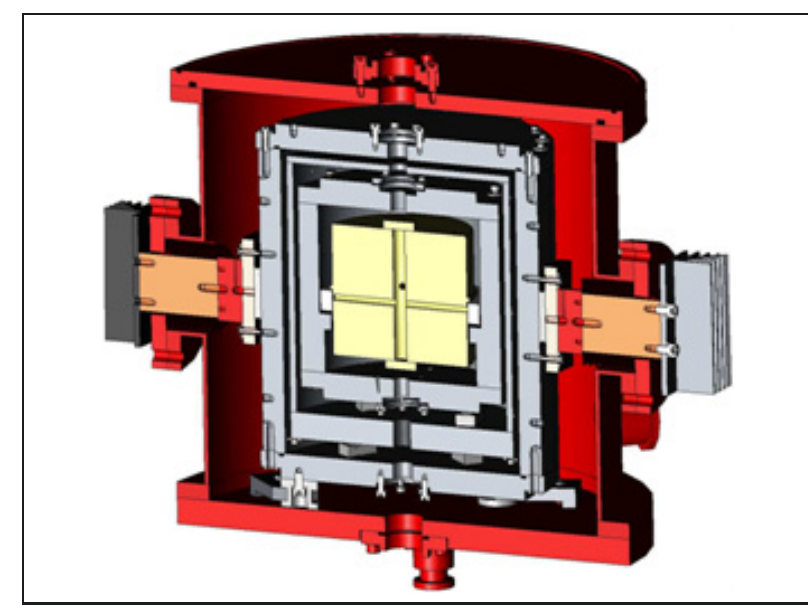

Fig. 13. - Cavité Fabry-Perot ultrastable servant à la stabilisation en fréquence du laser horloge à $1062,5 \mathrm{~nm}$.

de la mi-hauteur de la cavité, ont été optimisées au moyen de simulations numériques par éléments finis afin de minimiser la sensibilité accélérométrique [25]. Les substrats des miroirs de la cavité sont en silice fondue. Ce matériau permet de réduire les fluctuations de la longueur optique de la cavité liées au mouvement brownien des miroirs (ou bruit thermique) [26]. Les miroirs de la cavité ont un traitement diélectrique spécialisé de très haute réflectivité et de très faible perte. Nous avons mesuré une finesse de cavité de 850000 (soit une largeur de résonance de $1,7 \mathrm{kHz}$ ).

Comme montré sur la figure 13, la cavité est entourée d'un ensemble d'écrans thermiques indispensables pour réduire très fortement l'influence des variations de température. En partant de la cavité, on trouve deux écrans thermiques dorés, puis une enceinte à vide en aluminium stabilisée en température à l'aide de module Peltier. L'ensemble est placé dans une seconde enceinte à vide (en rouge). Entre l'enceinte stabilisée en aluminium et la cavité, nous avons mesuré des temps caractéristiques des échanges thermiques supérieurs à 4 jours. Grâce à ce filtrage passif très puissant, on peut obtenir des instabilités de température inférieures à $10 \mathrm{nK}$ pour la cavité sur des temps allant jusqu'à $1000 \mathrm{~s}$, ce qui est le paramètre le plus important pour la réalisation d'une horloge optique. Enfin, l'ensemble du dispositif est placé sur une plateforme antivibration passive du commerce et dans un caisson antibruit. L'ensemble cavité/écrans/enceinte à vide a servi de point de départ au développement, en partenariat avec un industriel et le CNES, d'une cavité à vocation spatiale et, à partir de cette cavité, d'un laser ultrastable transportable à $1,5 \mu \mathrm{m}$ [27].

La lumière ultrastable engendrée à l'intérieur du caisson antibruit, sur la plateforme anti-vibration est ensuite distribuée par fibres optiques. Pour éviter une dégradation de la stabilité de fréquence de la lumière ultrastable lors de sa propagation dans les fibres optiques (du fait des vibrations et des variations de température), il est nécessaire de stabiliser activement la longueur optique de chacune de ces fibres. Pour cela, un interféromètre hété- rodyne est réalisé à même la plateforme, afin de mesurer les fluctuations de longueur des fibres.

\subsection{Multiplication vers le domaine ultraviolet}

Pour atteindre la longueur d'onde de la transition horloge, la lumière ultrastable est doublée deux fois, comme cela est représenté sur la figure 14. Après passage dans l'interféromètre du système de stabilisation des fibres, la lumière ultrastable à $1062,5 \mathrm{~nm}$ est injectée dans une fibre optique allant vers la table optique principale de l'expérience. La lumière est décalée en fréquence au moyen d'un modulateur acousto-optique. Quelques centaines de microwatts sont ensuite utilisées pour injecter une diode laser DFB. L'injection optique permet de recopier la stabilité en fréquence du laser ultrastable, tout en augmentant considérablement la puissance, puisque la diode laser DFB délivre jusqu'à $250 \mathrm{~mW}$ de puissance utile, après l'isolation optique. Une petite fraction de la lumière émise par la diode laser DFB rebrousse chemin à travers le modulateur acousto-optique et la fibre, ce qui permet de faire fonctionner le système de correction active des fluctuations de la fibre, comme indiqué sur la figure 14 .

Le premier doublage est effectué avec une cavité en anneau de type «bow-tie » asservie en fréquence sur la lumière incidente. Un cristal doubleur à quasi-accord de phase de type PP-MgO :SLT est utilisé. On obtient jusqu'à $140 \mathrm{~mW}$ de lumière ultrastable à 531,2 nm. Le second doublage est réalisé avec une seconde cavité en anneau et un cristal de BBO taillé pour un accord de phase angulaire. On génère ainsi jusqu'à $7 \mathrm{~mW}$ de puissance à la longueur d'onde de la transition horloge de 265,6 nm. Enfin, la fréquence est décalée avec un modulateur acousto-optique qui est utilisé pour ajuster et pour pulser la puissance envoyée vers les atomes de mercure. Dans des phases préliminaires de l'expérience, ou pour réaliser certaines manipulations des atomes, on utilise toute la puissance disponible. Pour obtenir des largeurs de raie de $10 \mathrm{~Hz}$ pour la spectroscopie des atomes confinés dans le réseau, on utilise des puissances de l'ordre de $500 \mathrm{nW}$.

\subsection{Connexion aux peignes de fréquences optiques}

Le laser ultrastable à $1062,5 \mathrm{~nm}$ est connecté à d'autres références ultrastables et à d'autres horloges du laboratoire. Dans un premier temps, un peigne de fréquences optiques réalisé sur la base d'un laser femtoseconde de type titane:saphir a été utilisé. Ce système et le laser ultrastable à $1062,5 \mathrm{~nm}$ ont permis l'implémentation d'un peigne stabilisé de manière directe sur la référence optique [24], une configuration simple et efficace qui a ensuite été utilisée sur tous les peignes du LNE-SYRTE. Dans cette configuration, le peigne engendre un signal micro-onde ultrastable qui est comparé à la référence ultrastable du LNE-SYRTE fondée sur un oscillateur cryogénique à résonateur en saphir [28, 29]. 


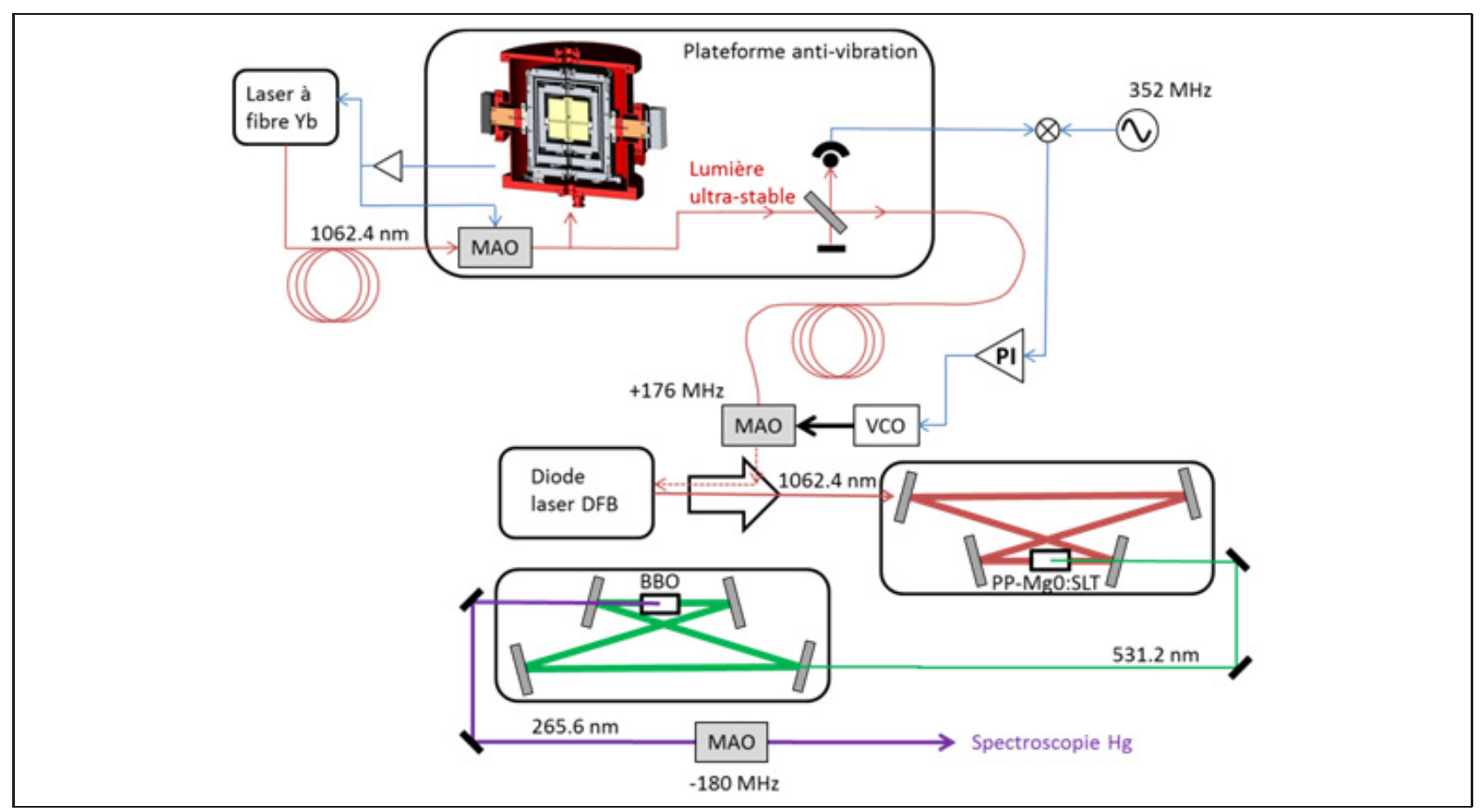

Fig. 14. - Schéma de distribution et de génération de la lumière ultrastable pour l'interrogation des atomes de mercure.

Dans un second temps, la connexion du laser ultrastable à $1062,5 \mathrm{~nm}$ a été réalisée avec un peigne de fréquences optiques sur un laser femtoseconde à fibre, beaucoup plus fiable et approprié pour des mesures fréquentes, longues ou permanentes. Le même peigne permet à la fois la connexion au domaine micro-onde et aux fontaines atomiques [29,30], la connexion à des références ultrastables à $1,5 \mu \mathrm{m}$ et les réseaux optiques fibrés, et la connexion vers les horloges à réseau optique à atomes de strontium. Pour plus de détails sur le peigne de fréquences à fibre, on pourra se reporter à la référence [3].

\subsection{Stabilité de la fréquence du laser ultrastable à $1062,5 \mathrm{~nm}$}

La figure 15 montre la stabilité de la fréquence entre le laser ultra-stable à $1062,5 \mathrm{~nm}$ et trois autres oscillateurs ultra-stables. La courbe rouge est une comparaison avec un second système à 1062,5 nm, basé sur une cavité horizontale. Les deux lasers étant à la même longueur, la mesure est faite de manière directe, à partir du battement entre les lasers. À une seconde, on observe une stabilité inférieure à $6 \times 10^{-6}$. On voit aussi que la stabilité est pratiquement constante sur une décade, ce qui est attendu pour une cavité limitée par le bruit thermique. Comme les deux cavités ont la même longueur, les mêmes substrats et les mêmes traitements diélectriques, il est raisonnable de supposer que chaque cavité contribue de manière égale. On peut ainsi estimer que la stabilité à une seconde de chacun des lasers vaut $4 \times 10^{-16}$, en bon accord avec ce que prévoient les modèles de bruit thermique [26]. Au-delà de quelques secondes, la stabilité est limitée par la cavité horizontale, qui n'avait pas de stabilisation de température.

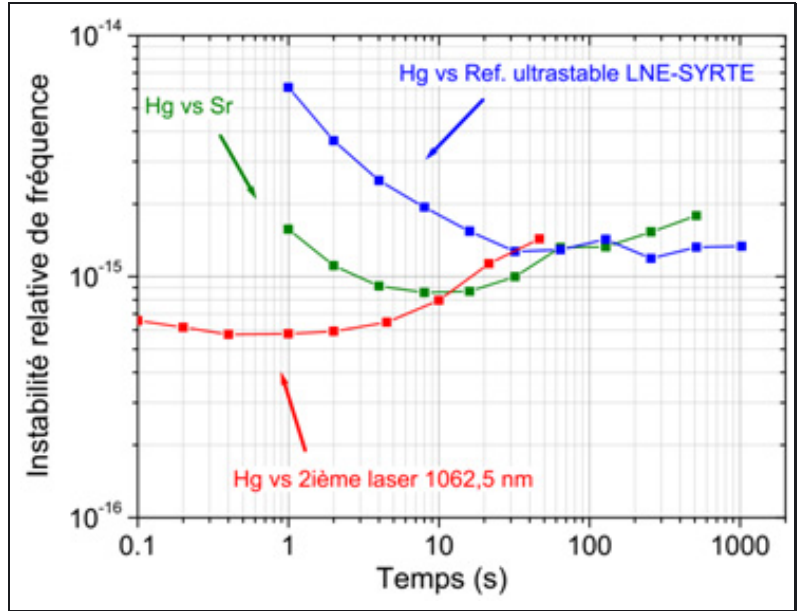

Fig. 15. - Stabilité de fréquence du laser ultrastable à $1062,5 \mathrm{~nm}$ par rapport à d'autres références ultrastables du LNE-SYRTE.

La courbe verte correspond à une comparaison avec le laser ultrastable à $698 \mathrm{~nm}$ des horloges à réseau optique de strontium. La mesure est effectuée via le peigne de fréquence titane:saphir dont le bruit limite la mesure à $1 \mathrm{~s}$. Autour de $10 \mathrm{~s}$, la stabilité est proche de la limite déterminée par les bruits thermiques des deux cavités.

La courbe bleue correspond à une comparaison avec la référence ultrastable du LNE-SYRTE. La mesure est effectuée via le peigne de fréquence titane:saphir stabilisé sur le laser à $1062,5 \mathrm{~nm}$. Le signal micro-onde ultrastable généré par le peigne est comparé au signal micro-onde à 9,2 GHz issu de la référence ultrastable du LNE-SYRTE. La courbe bleue montre la stabilité après 
soustraction de la dérive linéaire stable et prédictible observée sur toutes les cavités de ce type et qui est due au fluage du verre constitutif de la cavité. Sur le laser à $1062,5 \mathrm{~nm}$, on observe une dérive particulièrement faible de $-6 \times 10^{-17} \mathrm{~s}^{-1}$, soit $\left(-17 \mathrm{mHz} \cdot \mathrm{s}^{-1}\right)$ La stabilité à une seconde est limitée par le lien par fibre à $1 \mathrm{GHz}$ transmettant la référence ultrastable au peigne de fréquences. La stabilité à $1000 \mathrm{~s}$ est inférieure à $2 \times 10^{-15}$, et on peut estimer qu'il y a des contributions égales du laser et de la référence ultrastable. Ce bon comportement du laser à $1062,5 \mathrm{~nm}$, sur des durées assez longues, vient notamment de l'efficacité du filtrage passif produit par le système d'écrantage thermique.

\section{Spectroscopie de la transition horloge et détermination de la longueur d'onde magique}

Nous décrivons les expériences qui ont permis la réduction préalable des incertitudes importantes qui affectaient deux quantités clés pour l'horloge à réseau optique : la fréquence même de la transition et la longueur d'onde magique.

\subsection{Spectroscopie préliminaire sur les atomes froids en chute libre}

$\mathrm{Au}$ démarrage du développement de l'horloge à atomes de mercure, la spectroscopie laser de la transition ${ }^{1} \mathrm{~S}_{0-}{ }^{3} \mathrm{P}_{0}$ du mercure n'avait jamais été faite. La fréquence de cette transition était connue de manière indirecte à partir de mesures effectuées sur des lampes à vapeur de mercure [31-35], avec des incertitudes d'environ $500 \mathrm{MHz}$, sauf pour l'isotope ${ }^{198} \mathrm{Hg}$ pour lequel l'incertitude était de $12 \mathrm{MHz}$

La première spectroscopie laser de la transition horloge a été effectuée sur les atomes froids issus du PMO. Elle a été effectuée sur les deux isotopes fermioniques ${ }^{199} \mathrm{Hg}$ et ${ }^{201} \mathrm{Hg}$ pour lesquels la transition est faiblement autorisée par l'interaction hyperfine. Lors de la phase de recherche initiale des transitions, le PMO était allumé de manière continue, jusqu'à atteindre son état stationnaire. Un faisceau d'interrogation vertical de quelques milliwatts (puissance maximum disponible), lui aussi continu, était envoyé à travers le PMO. Dans ces conditions, lorsque la résonance avec la transition horloge est atteinte, une fraction des atomes est transférée vers l'état métastable ${ }^{3} \mathrm{P}_{0}$ où ils deviennent insensibles au piégeage magnéto-optique, accélèrent sous l'effet de la gravité, se désaccordent du faisceau d'interrogation par effet Doppler et quittent finalement la zone du PMO. Cette interaction crée ainsi un mécanisme de perte d'atomes qui diminue le nombre d'atomes et la fluorescence du PMO en régime stationnaire. Dans les conditions de notre expérience et malgré le faible couplage de la transition horloge, il s'avère que ce mécanisme pouvait causer une déplétion du PMO de $90 \%$. La figure 16a montre un des premiers signaux de spectroscopie laser directe de la transition ${ }^{1} \mathrm{~S}_{0}-{ }^{3} \mathrm{P}_{0}$ de l'isotope ${ }^{199} \mathrm{Hg}$ obtenus selon cette méthode.

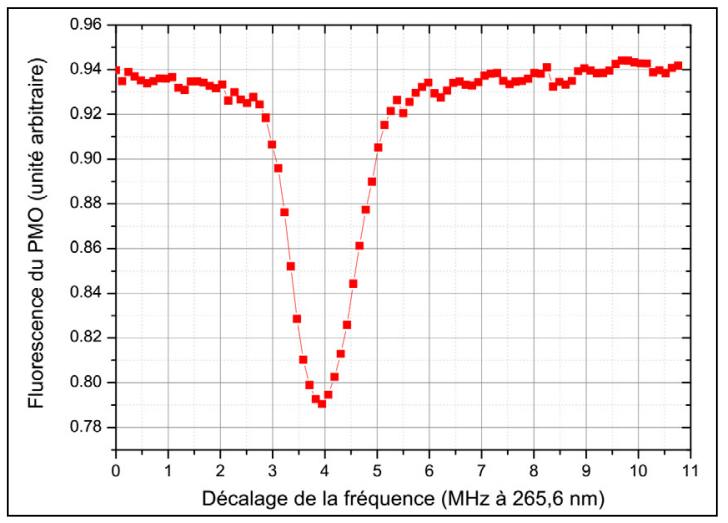

(a)

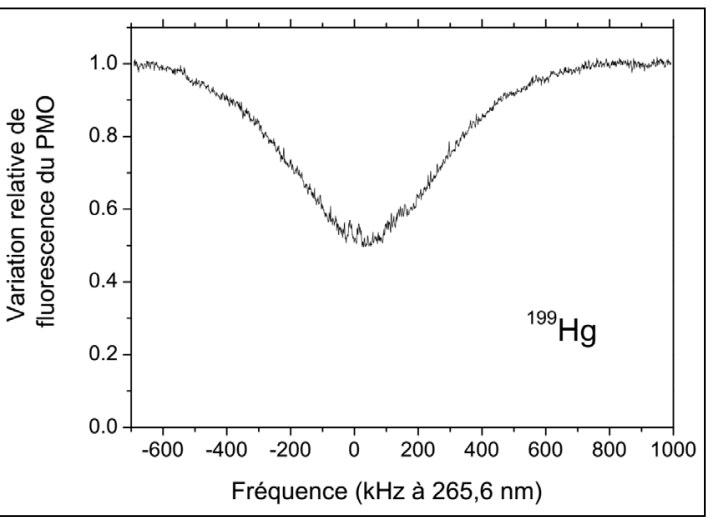

Fig. 16. - Spectres de la transition horloge ${ }^{1} \mathrm{~S}_{0}-{ }^{3} \mathrm{P}_{0}$ de ${ }^{199} \mathrm{Hg}$ observé par les pertes induites sur un piège magnéto-optique à l'état stationnaire. Les graphes montrent l'amplitude de fluorescence stationnaire du piège en fonction de la fréquence du décalage en fréquence du laser d'excitation. (a) avec un faisceau d'interrogation continu, lors de la recherche initiale de la transition. (b) avec un faisceau d'interrogation pulsé.

Ces conditions d'expérience sont appropriées à la recherche initiale mais pas à une mesure de bonne qualité de la fréquence de la transition. En effet, l'excitation y est notamment affectée par le déplacement lumineux liée à la lumière du piège, qui est probablement la cause de l'asymétrie visible sur la figure 16a. Pour éviter cet effet, une séquence pendant laquelle les faisceaux du PMO sont allumés pendant $50 \mathrm{~ms}$ puis éteints pendant $5 \mathrm{~ms}$, durant lesquelles le faisceau d'interrogation est allumé. Sous l'effet d'une telle séquence, le PMO atteint un état stationnaire qui, comme précédemment, est affecté par les pertes induites par le laser d'interrogation. Par ailleurs, le faisceau d'interrogation est rétro-réfléchi. Cette configuration permet de réduire d'observer les pics de saturation qui ont la propriété d'être insensibles à l'effet Doppler. La figure 16 b montre exemple de spectre observé avec cette nouvelle séquence. Le spectre est plus symétrique et plus étroit, du fait de la suppression du déplacement lumineux. Comme on l'a déjà mentionné, sa largeur donne une bonne indication de la température des atomes. À proximité de l'extremum, on distingue deux pics étroits qui sont les pics de saturation.

La figure 17 montre les pics de saturation pour les deux isotopes fermioniques. Le spectre prend la forme 


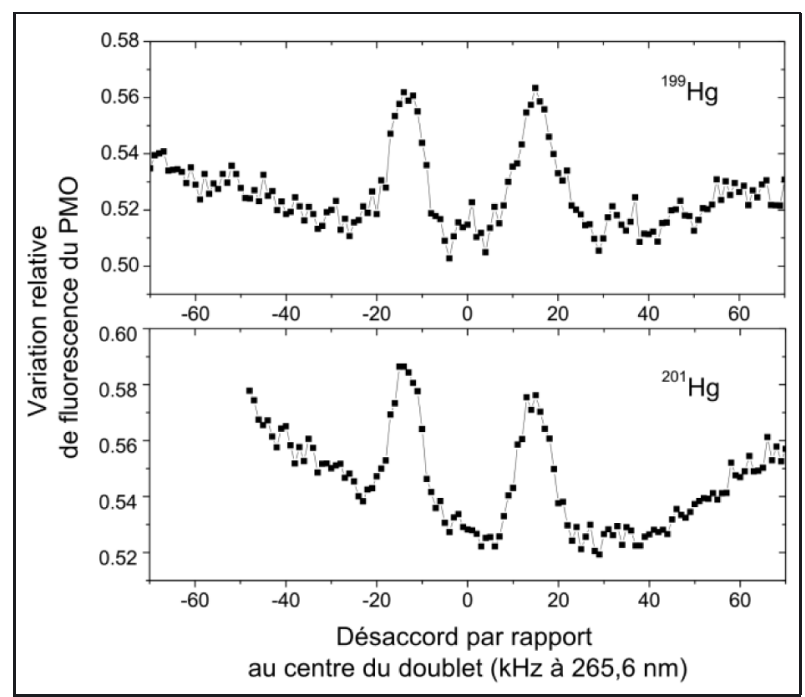

Fig. 17. - Doublets de recul de la transition horloge ${ }^{1} \mathrm{~S}_{0}{ }^{3} \mathrm{P}_{0}$ de ${ }^{199} \mathrm{Hg}$ et ${ }^{201} \mathrm{Hg}$ observée sur des atomes lâchés en chute libre depuis un piège magnéto-optique.

d'un doublet appelé doublet de recul, dont l'espacement est égal au double de la fréquence de recul $v_{R}=v \times$ $\left(h v / 2 m c^{2}\right)=14,2 \mathrm{kHz}$, où $v$ est la fréquence de la transition, $m$, la masse atomique, $h$, la constante de Planck et $c$ la vitesse de la lumière. Cette fréquence correspond à l'énergie de recul (énergie cinétique qu'acquière un atome au repos) lorsqu'il absorbe un unique photon du laser d'interrogation. La fréquence des pics du doublet de recul n'est pas affectée par l'effet Doppler et le centre du doublet de recul coöncide avec la fréquence atomique non perturbée. Nous avons étudié les propriétés du doublet de recul en fonction des divers paramètres de la séquence d'interrogation et estimer les sources de déplacements de la fréquence de la transition [36]. Enfin, grâce à la connexion aux références primaires du LNE-SYRTE basées sur les fontaines atomiques, nous avons déterminé, pour les deux isotopes fermioniques, la fréquence absolue de la transition hor-

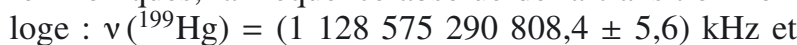
$v\left({ }^{201} \mathrm{Hg}\right)=\left(\begin{array}{l}1 \\ 1\end{array} 28569561139,6 \pm 5,3\right) \mathrm{kHz}$. Les incertitudes ainsi obtenues représentent une amélioration de plus de quatre ordres de grandeurs par rapport au niveau de connaissance précédent.

\subsection{Réseau optique pour le piégeage du mercure}

Nous avons décrit le principe et l'utilité du piégeage dans un réseau optique rendu «non-perturbateur » grâce au choix précis de la longueur d'onde du piège. Une condition indispensable à la réalisation d'une horloge à réseau optique est donc l'existence même d'une longueur d'onde pour laquelle les polarisabilités des deux états horloges sont identiques. Il faut également, qu'à cette même longueur d'onde, l'émission spontanée induite par le laser intense du réseau soit très faible, ce qui impose que la longueur d'onde soit très éloignée de toutes les longueurs d'onde des transitions atomiques impliquant les niveaux énergétiques horloges. Le choix de l'atome de mercure a bien sûr été fait après une analyse des pro- priétés atomiques établissant l'existence d'une longueur d'onde magique convenable. La valeur de la longueur d'onde magique n'était alors estimée que de manière théorique, avec des incertitudes élevées et mal déterminées, notamment au regard du choix et du développement des systèmes lasers et optoélectroniques adéquats. Ainsi, les estimations théoriques publiées vont de $342 \mathrm{~nm}$ à $365 \mathrm{~nm}$. Prédire une longueur d'onde magique avec une incertitude de quelques nanomètres nécessite la mise en œuvre des méthodes de calcul de physique atomique les plus poussées.

Une étape essentielle du développement d'une horloge à réseau optique fondée sur un atome jusqu'alors inexploré est donc de déterminer expérimentalement la valeur de la longueur d'onde magique. Nous avons choisi de réaliser cette étape directement à partir de la spectroscopie d'atomes piégés dans un réseau optique. Les figures 18 et 19 montrent les systèmes permettant ce piégeage, c'est-à-dire la source laser d'une part et, d'autre part, la cavité Fabry-Perot sous ultravide dont le mode traverse le nuage atomique.

Comme indiqué sur la figure 18, la source laser pour le réseau optique est réalisée par doublage d'un laser titane:saphir, pompé à $532 \mathrm{~nm}$ par un laser $\mathrm{Nd}: \mathrm{YVO}_{4}$ doublé et pompé par diode commercial. Il s'agit d'un système de laboratoire préexistant, qui a été modifié pour atteindre les longueurs d'onde courtes nécessaires ici. La géométrie de la cavité laser est de type en anneau nonplanaire [37]. Le choix de ce laser a été fait considérant qu'il permettait, pour la phase d'exploration, d'atteindre une bonne partie de la gamme la plus probable pour la longueur d'onde magique. La valeur de la longueur d'onde magique étant confirmée, on pourrait maintenant opter pour un laser du commerce, sachant qu'il en existe maintenant de très fiables et puissants. La fréquence de la lumière issue du laser titane:saphir est doublée avec un cristal de LBO, taillé à l' angle de Brewster et accordé angulairement. La cavité de doublage en anneau est asservie sur la fréquence de la lumière incidente avec une détection de type Hänsch-Couillaud. La puissance générée par cette source dépend fortement de la longueur d'onde du fait des caractéristiques du laser titane:saphir. Nous avons obtenu une puissance jusqu'à $280 \mathrm{~mW}$. Dans les conditions usuelles récentes, on génère typiquement une puissance de $140 \mathrm{~mW}$ à la longueur d'onde magique. La lumière issue du doublage passe à travers un modulateur acousto-optique qui permet, pour certaines expériences, de soustraire une partie de la puissance du faisceau principal et de moduler ainsi sa puissance. La lumière est ensuite injectée dans la cavité Fabry-Perot du réseau optique. Comme indiqué sur la figure 18, la lumière transmise par la cavité, extraite par un miroir dichroïque permettant de superposer le faisceau laser d'interrogation (à 265,6 nm), est détectée par une photodiode. Après soustraction d'une valeur de référence, on réalise un asservissement à $90 \%$ du sommet du pic de transmission. Le premier étage de l'asservissement asservit la fréquence du laser titane:saphir sur la fréquence de la cavité. Un second étage intégral agit lentement sur les cales piézoélectriques de la cavité Fabry-Perot pour maintenir 


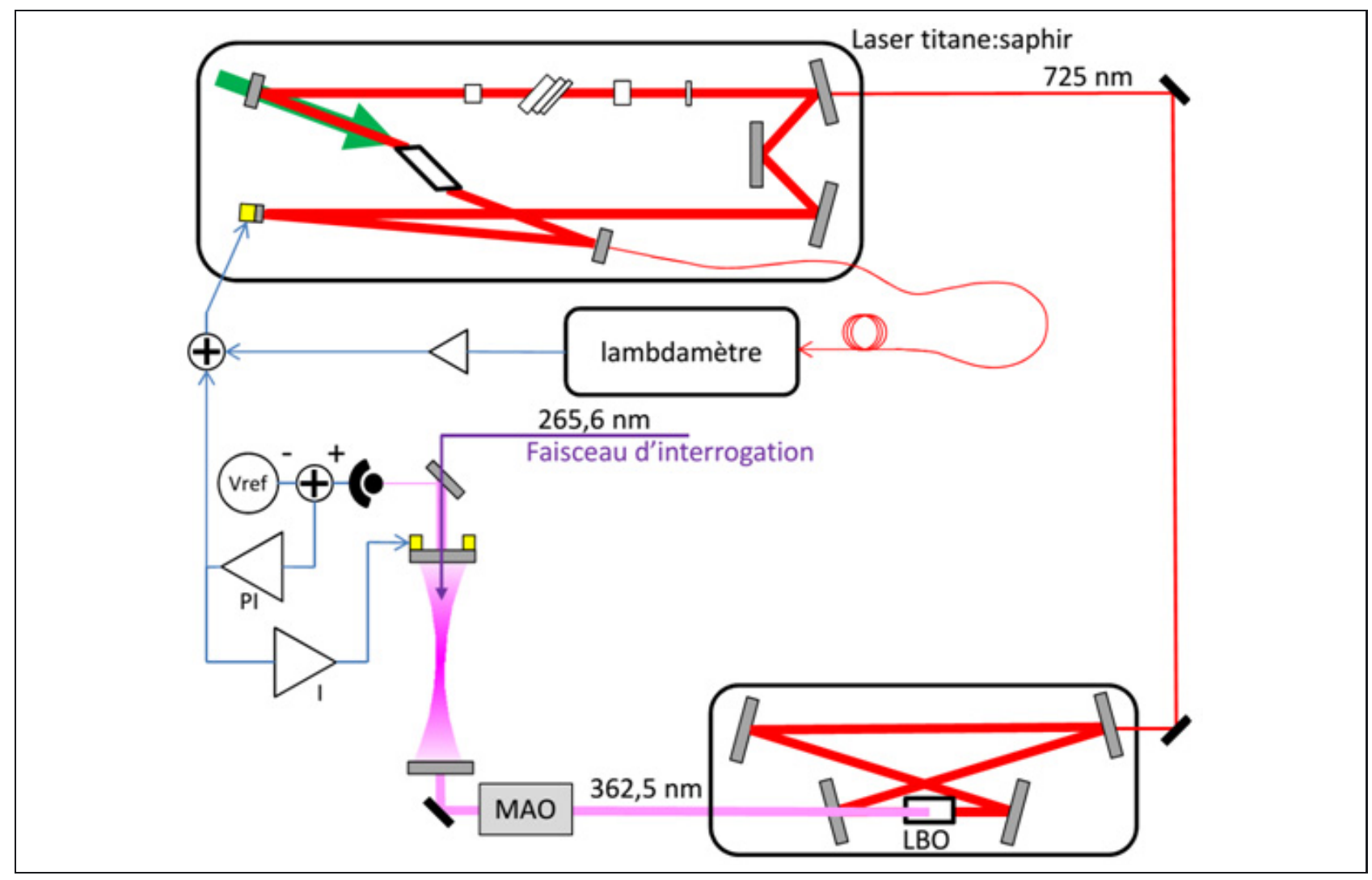

Fig. 18. - Système laser pour le piégeage des atomes de mercure dans un réseau optique à la longueur d'onde magique.

la correction appliquée au laser titane:saphir proche de zéro. Afin, la stabilisation de l'ensemble sur le long terme peut être réalisée via un système numérique à partir de la mesure faite par un lambdamètre haute résolution.

La figure 19 est une représentation tridimensionnelle de la cavité Fabry-Perot formant le réseau optique. La cavité est verticale. Les deux miroirs de la cavité servent en même temps de fenêtres de l'enceinte à ultravide. Ce choix permet d'éviter toute interface supplémentaire créant des pertes optiques à l'intérieur de la cavité. Ainsi, on obtient des finesses et donc des surtensions élevées, ce qui est indispensable, dans le cas du mercure, pour obtenir une puissance circulante et une profondeur de piège suffisamment élevée. Un autre avantage de la cavité entièrement sous ultravide est que la lumière intense reste très bien confinée et génère très peu de lumière parasite dans le système de détection. Dans la dernière mise en œuvre de la cavité, nous avons une finesse de 360 et le mode de la cavité forme un «waist » de $69 \mu \mathrm{m}$ au niveau du nuage atomique. Dans ces conditions, avec une puissance circulante de $10 \mathrm{~W}$ dans la cavité, on obtient une profondeur de piège de $37 \mu \mathrm{K}$, ce qui correspond environ à 100 fois l'énergie de recul $E_{\mathrm{R}}$ à la longueur d'onde du piège. Dans les premières expériences, nous étions limités à des profondeurs beaucoup plus modestes (10 énergies de recul), du fait d'un waist plus grand et d'une dégradation des optiques de la cavité.

Une étape importante a été l'obtention et la caractérisation du piégeage dans le réseau optique. Le réseau

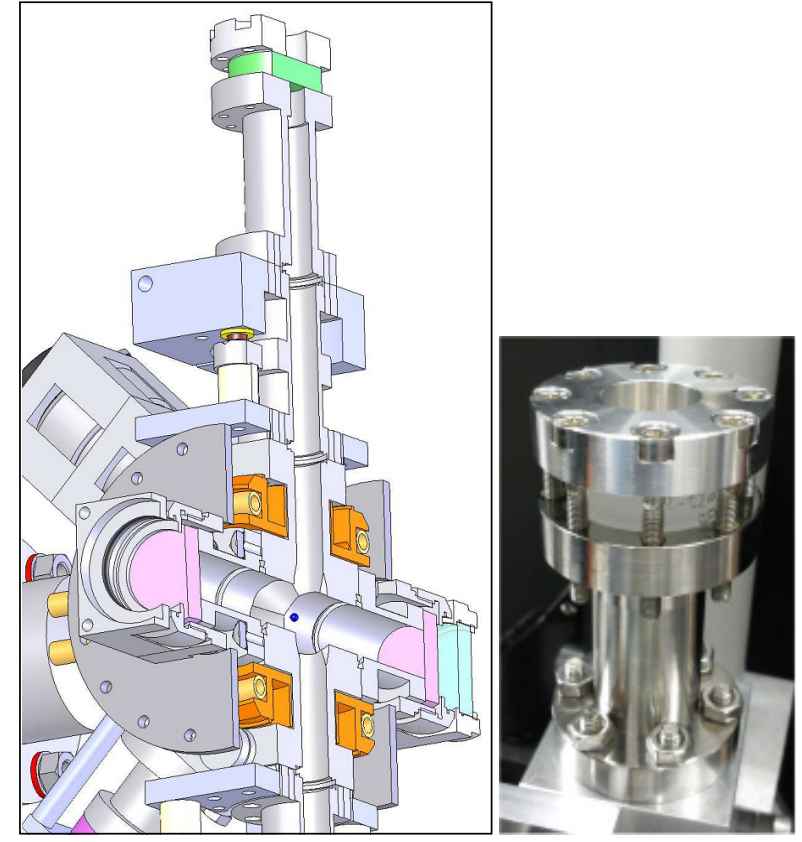

Fig. 19. - Vue en coupe de l'enceinte à vide montrant la cavité Fabry-Perot sous ultravide pour le piégeage des atomes de mercure dans un réseau optique à la longueur d'onde magique. Un des deux miroirs de la cavité est représenté en vert, en haut de la figure. La petite sphère en bleu foncé au centre signale la position du nuage atomique. La cavité est symétrique par rapport au nuage atomique. La photographie montre un des miroirs de la cavité assemblé sur une pièce en titane à l'aide d'un joint en indium, pendant la phase de préparation et de test. 


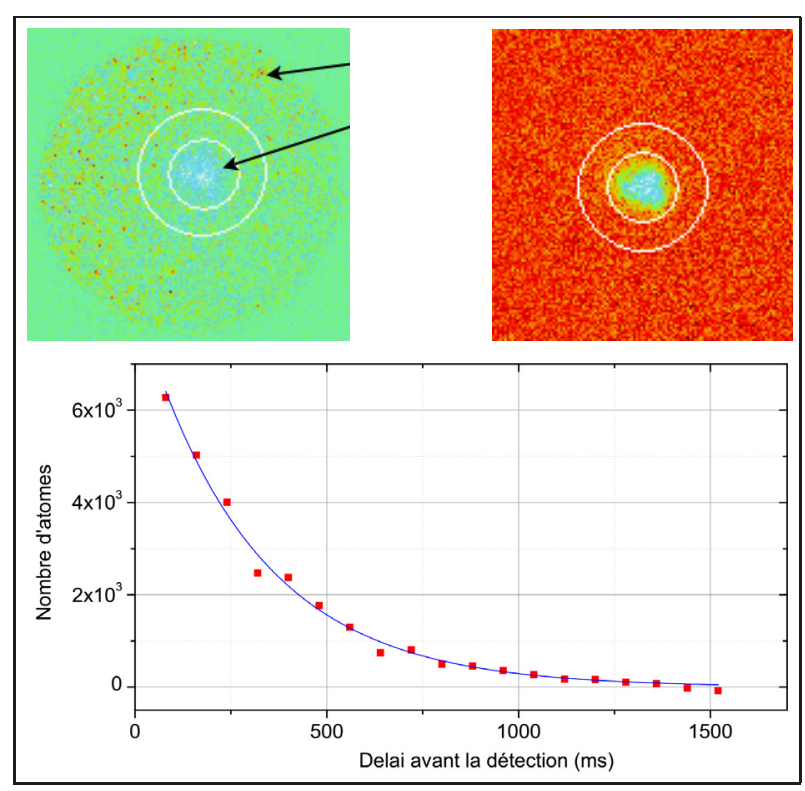

Fig. 20. - En haut à gauche : une des premières images des atomes de mercure piégés dans un réseau optique d'une profondeur proche de 10 énergies de recul $E_{\mathrm{R}}$. En haut à droite : une image récente pour un piège d'une profondeur proche de $50 E_{\mathrm{R}}$. Le nombre d'atomes y est d'environ $10^{4}$, soit 10 fois plus que dans la première image. En bas : mesure de la durée de vie des atomes dans le piège.

optique est gardé allumé continument, traversant le PMO, ce qui requiert des étapes d'alignements délicates. Après le chargement du PMO, les atomes sont refroidis de manière optimale, comme déjà décrit. Les faisceaux lasers sont alors coupés brusquement (typiquement en $100 \mu$ s). Les atomes suffisamment lents et situés aux niveaux des extrema d'intensité du réseau optique restent piégés, alors que les autres atomes tombent et s'éloignent balistiquement. Après un délai ajustable selon les expériences, on peut faire l'image des atomes restés piégés dans le réseau, en utilisant le système décrit plus haut. La fraction d'atomes restant dans le réseau dépend de la taille et de la température du PMO, et donc de l'isotope, de la taille et de la profondeur du réseau optique, et de la qualité de l'alignement. Lorsque le chargement a été recherché et obtenu pour la première fois, la fraction capturée était limitée à environ $10^{-3}$ et le nombre d'atomes détectés de seulement quelques centaines. Dans les mesures les plus récentes, nous avons observé jusqu'à $10^{4}$ atomes de ${ }^{199} \mathrm{Hg}$. La figure 20 montre une des premières images des atomes piégés dans le réseau, où l'on peut constater que le signal est plutôt ténu, ainsi qu'une image récente. Le graphe de la figure 20 montre une mesure du nombre d'atomes resté dans le réseau en fonction du délai avant la détection. La durée de vie des atomes dans le réseau est ici de $300 \mathrm{~ms}$.

\subsection{Détermination de la longueur d'onde magique du mercure}

Pour réaliser la spectroscopie de la transition horloge dans le réseau, on implémente une séquence où l'on

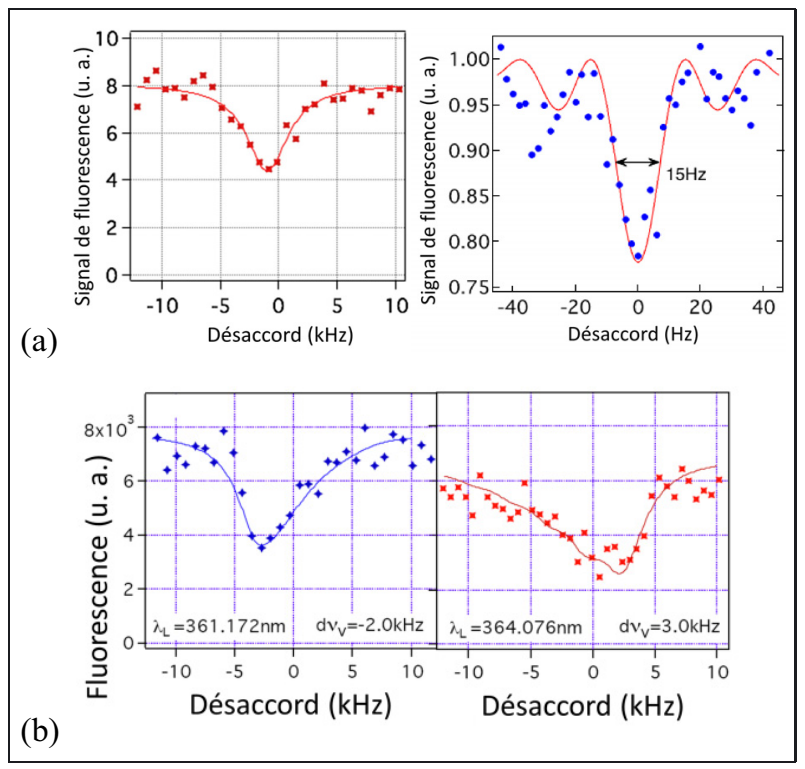

Fig. 21. - Spectroscopie de la transition horloge dans le réseau optique. (a) gauche : Un des premiers spectres obtenus, lorsque la longueur d'onde du réseau est peu éloignée de la longueur d'onde magique. La largeur de raie est de $4 \mathrm{kHz}$; (a) droite : un spectre obtenu après plusieurs améliorations du dispositif. La largeur de raie est de $15 \mathrm{~Hz}$; (b) deux exemples de spectres mesurés lorsque la longueur d'onde du réseau est éloignée de la longueur d'onde magique. Dans ce cas, l'asymétrie des spectres devient manifeste.

détecte le nombre d'atomes dans le réseau après un délai fixe, selon les méthodes décrites ci-dessus. Pendant le délai entre l'extinction du PMO et la détection, on interpose l'interaction avec un faisceau de lumière ultrastable colinéaire à l'axe du réseau optique, comme cela est représenté sur les figures 2 et 18. Lorsque la résonance est atteinte, une fraction des atomes passe de ${ }^{1} \mathrm{~S}_{0}$ à ${ }^{3} \mathrm{P}_{0}$. Les atomes dans ${ }^{3} \mathrm{P}_{0}$ restent piégés par le réseau optique, mais ils ne sont pas détectés par le système de détection, uniquement sensible aux atomes dans le niveau ${ }^{1} \mathrm{~S}_{0}$. On observe donc la transition horloge par une diminution du nombre d'atomes détectés.

La figure 21 montre des exemples de spectre. Lors des premières expériences, la profondeur du réseau optique était très modeste $\left(<10 E_{\mathrm{R}}\right)$. Les largeurs de raie, dans ces conditions étaient typiquement de $4 \mathrm{kHz}$. À partir d'une série de spectres mesurées pour deux profondeurs différentes $\left(10,1 E_{\mathrm{R}}\right.$ et $\left.6,8 E_{\mathrm{R}}\right)$ et pour plusieurs valeurs de la longueur d'onde du réseau, nous avons déterminé le déplacement de fréquence de la transition, en fonction de la longueur d'onde. La figure 22 montre de graphe correspondant. La sensibilité du déplacement lumineux à un changement de la longueur d'onde du réseau optique (exprimée en fréquence) est estimée à $5 \times 10^{-17} E_{\mathrm{R}}^{-1} \mathrm{GHz}^{-1}$, ce qui est conforme à la valeur calculée et très proche des valeurs de $\mathrm{Sr}$ et $\mathrm{Yb}$. Ces mesures donnent la première détermination expérimentale de la longueur d'onde magique pour l'atome de mercure ${ }^{199} \mathrm{Hg}: 362,53 \pm 0,21 \mathrm{~nm}$. La figure 21 montre des spectres obtenus lorsque la longueur d'onde du réseau est éloignée de la longueur 


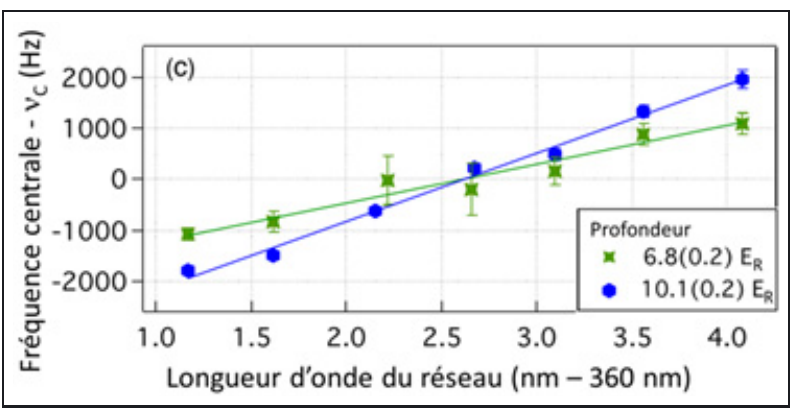

Fig. 22. - Mesures du déplacement de la fréquence en fonction de la longueur d'onde du réseau optique pour deux profondeurs de piège. Cette série de mesures a donné la première détermination expérimentale de la longueur d'onde magique pour l'atome de mercure ${ }^{199} \mathrm{Hg}$.

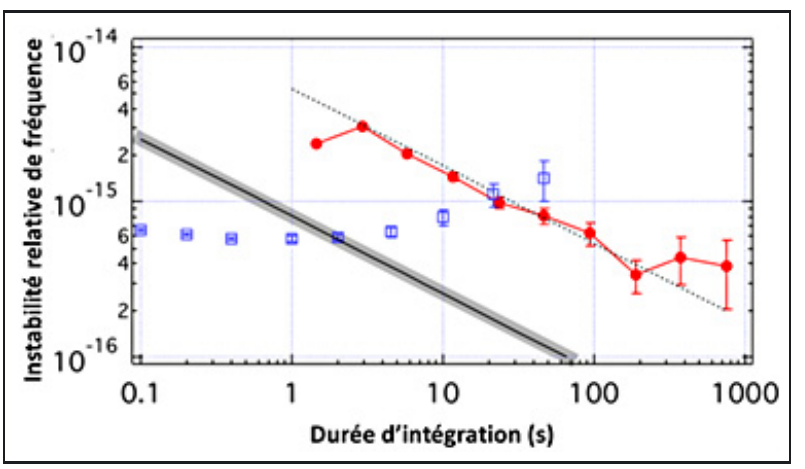

Fig. 23. - Stabilité à court terme de l'horloge ${ }^{199} \mathrm{Hg}$, en rouge. Les points bleus reproduisent la comparaison entre le laser ultrastable à $1062,5 \mathrm{~nm}$ et l'autre système à $1062,5 \mathrm{~nm}$ de la figure 15. La zone grisée représente l'estimation de la limite de stabilité de l'horloge liée au seul bruit du laser ultrastable.

d'onde magique. L'élargissement asymétrique lié à la différence de déplacement lumineux entre les différents niveaux vibrationnels devient manifeste. Une analyse de ces asymétries à partir d'un modèle donne une autre méthode de détermination de la longueur d'onde magique, en bon accord avec la première $[21,38]$. Dans un second temps, des améliorations ayant donné accès à des pièges plus profonds (jusqu'à $24 E_{\mathrm{R}}$ ) et à des largeurs de raie beaucoup plus étroites, la détermination de la longueur d'onde magique a été grandement améliorée : $\lambda_{\text {piège }}=362,5697 \pm 0,0011 \mathrm{~nm}[39]$.

\section{Mesures absolues de la fréquence et valeur recommandée pour la transition horloge de ${ }^{199} \mathrm{Hg}$}

À la longueur d'onde magique et pour des pièges les plus profonds que l'on ait pu obtenir, nous avons obtenu des largeurs allant jusqu'à $11 \mathrm{~Hz}$. La fréquence de la transition horloge correspondant à la longueur d'onde de 265,6 nm est $1128 \mathrm{THz}$. Cela correspond donc à un facteur de qualité atomique $Q_{a t}=10^{14}$. Lorsque l'on asservit la fréquence du laser d'interrogation sur la transition atomique et qu'on analyse les corrections de fréquence, on peut déterminer la stabilité à court terme de la fréquence de l'horloge. La figure 23 montre un exemple d'une telle mesure. On obtient une stabilité à court terme de $5,4 \times 10^{-15} \tau^{-1 / 2}$. Cette valeur reste limitée par les conséquences de la faible profondeur du réseau optique (nombre d'atomes modestes, raie imparfaitement contrastée) et par des fluctuations d'origine technique du nombre d'atomes détecté. La limite liée au bruit du laser d'interrogation à $1062,5 \mathrm{~nm}$ est inférieure à $10^{-15} \tau^{-1 / 2}$, comme cela est décrit dans la référence [40]. Comme on le voit sur la figure 23 , on peut effectuer une mesure avec une résolution de quelques $10^{-16}$, soit une fraction de hertz en quelques minutes.

Nous avons réalisé une série de mesures avec cette valeur typique de résolution afin de réaliser une première évaluation des principaux effets systématiques. Nous avons observé et mesuré les transitions Zeeman de la transition horloge de ${ }^{199} \mathrm{Hg}$. Nous avons mesuré l'effet Zeeman du second ordre pour des champs magnétiques de l'ordre de $10 \mu \mathrm{T}$. Le déplacement relatif du second ordre attendu est de $-2,2 \times 10^{-21} \mu \mathrm{T}^{-2}$, soit $-2,2 \times 10^{-19}$ pour $10 \mu \mathrm{T}$. Conformément à cette estimation, à la résolution que nous pouvons atteindre, nous avons constaté une absence de dépendance quadratique. Nous avons également mesuré le déplacement de la fréquence en fonction du nombre d'atomes piégés dans le réseau pour des nombres d'atomes allant jusqu'à $2,5 \times 10^{3}$, ce qui correspond à une densité moyenne estimée de $3 \times 10^{5} \mathrm{~mm}^{-3}$ et à environ 1,5 atomes par site du réseau optique. Nous avons constaté une absence de dépendance et placé une borne supérieure au déplacement collisionnel au niveau de $5 \times 10^{-21} \mathrm{~mm}^{3}$. Nous avons testé ou estimé le déplacement de fréquence lié au déplacement lumineux de la sonde.

Nous avons mesuré la température en plusieurs points de l'enceinte à vide en vue directe des atomes pour estimer que l'incertitude sur la température absolue du rayonnement vu par les atomes était de $(290 \pm 2) \mathrm{K}$. La correction de fréquence correspondante vaut $1,6 \times 10^{-16}$. L'incertitude liée à la température est de seulement $4 \times$ $10^{-18}$. On voit là tout l'intérêt de la faible sensibilité du mercure au rayonnement thermique. À cette incertitude, il faut ajouter l'incertitude liée au coefficient $\Delta \alpha_{a t}$ (et $\eta$ ) qui détermine le déplacement. Actuellement, le coefficient vient d'une estimation théorique qui malheureusement ne comporte pas d'estimation d'incertitude [41]. Une approche très prudente que nous avions adoptée dans [39], et qui est reproduite dans le tableau 2, consiste à considérer que l'incertitude est égale à la valeur calculée. Lorsqu' on examine l'historique de ce type de calcul et son niveau d'accord avec l'expérience pour d'autres atomes $[18,19,42]$, on peut estimer qu'une incertitude réelle est plutôt égale à $10 \%$ de la valeur, ce qui correspondrait à $1,6 \times 10^{-17}$. Enfin, nous avons mesuré le déplacement lumineux lié au piégeage par le réseau pour un ensemble de valeur de la profondeur et de la longueur d'onde du piège. Cela conduit, comme nous l'avons déjà mentionné, à une détermination de la longueur d'onde magique améliorée par rapport à la détermination initiale.

L'incertitude pour l'horloge est alors reliée à l'incertitude de la détermination et du contrôle de la longueur 
Tableau 2

Corrections et incertitudes de type $\mathrm{B}$ de l'horloge à réseau optique à atomes de ${ }^{199} \mathrm{Hg}$.

\begin{tabular}{|c|c|c|}
\hline $\begin{array}{c}\text { Composante } \\
\text { d'incertitude (effet } \\
\text { sur la fréquence) }\end{array}$ & Correction (Hz) & $\begin{array}{c}\text { Incertitude } \\
(\mathbf{H z})\end{array}$ \\
\hline Rayonnement thermique & 0,17 & 0,2 \\
\hline Zeeman ordre 1 & 0,0 & 0,5 \\
\hline Zeeman ordre 2 & 0,0 & 1,6 \\
\hline $\begin{array}{c}\text { Déplacement } \\
\text { lumineux du réseau }\end{array}$ & 0,0 & 5,9 \\
\hline $\begin{array}{c}\text { Déplacement } \\
\text { lumineux de la sonde }\end{array}$ & $-0,02$ & 0,3 \\
\hline $\begin{array}{c}\text { Effets de la densité } \\
\text { atomique }\end{array}$ & $-0,4$ & 1,9 \\
\hline $\begin{array}{c}\text { Incertitude de type B totale } \\
\text { (somme quadratique) }\end{array}$ & $\mathbf{6 , 4}$ \\
\hline
\end{tabular}

d'onde magique, à l'amplitude possible d'exploration de la profondeur du piège et à la profondeur du piège en fonctionnement. Au final, cette incertitude domine, et elle est elle-même limitée par la gamme relativement faible de profondeur de piège accessible $\left(<24 E_{\mathrm{R}}\right)$, par le degré de contrôle de la longueur d'onde du réseau et par la résolution typique de chaque mesure étant donné la précarité du fonctionnement de la source laser de refroidissement. Le tableau 2 résume les incertitudes et donne l'incertitude totale (de type B) qui vaut $5,7 \times 10^{-15}$. Quelques effets ne sont pas formellement inclus car on peut raisonnablement les considérer négligeables au niveau actuel, à la fois en déplacement et en incertitude. S'agissant d'un éventuel déplacement qui serait lié à un champ électrique statique parasite [17], on notera que la configuration de l'enceinte à ultravide (fig. 7 et 19) et l'exposition permanente à la lumière ultraviolette sont favorable à l'annulation du champ électrique statique au niveau des atomes. Au regard des investigations existantes dans d'autres systèmes, on peut estimer qu'au niveau actuel, l'effet et l'incertitude liés à un éventuel résidu de champ électrique statique sont négligeables devant ceux liés du rayonnement thermique.

Nous avons réalisé une série de mesures absolues de la fréquence de l'horloge à réseau optique ${ }^{199} \mathrm{Hg}$, via les peignes femtosecondes et par rapport à la référence de fréquence ultrastable du LNE-SYRTE, elle-même mesurée par rapport à l'étalon primaire en fontaine FO2Cs [29] dont l'exactitude est de $2,7 \times 10^{-16}$. La figure 24 montre cette série de mesures avec leurs incertitudes statistiques (de type A). On y voit que les mesures sont bien reproductibles. La moyenne pondérée de cet ensemble de mesure a une incertitude de type A de $2,5 \times 10^{-16}$. La fréquence de la transition horloge ${ }^{1} \mathrm{~S}_{0}{ }^{3} \mathrm{P}_{0}$ de ${ }^{199} \mathrm{Hg}$ vaut (1 $128575290808162,0 \pm 6,4$ (syst) $\pm 0,3) \mathrm{Hz}$. L'incertitude relative totale vaut $5,7 \times 10^{-15}$, ce qui représente une amélioration d'un facteur 875 par rapport à notre mesure préliminaire rapportée plus haut. Le résultat de cette mesure a été soumis au Comité Consultatif du Temps et des Fréquences (CCTF) du Comité International des Poids et Mesures (CIPM) lors de sa $19^{\mathrm{e}}$ réunion en septembre 2012. À l'issue de cette réunion, la transition ${ }^{1} \mathrm{~S}_{0^{-}}{ }^{3} \mathrm{P}_{0}$ de ${ }^{199} \mathrm{Hg}$ a été incluse dans la liste des valeurs

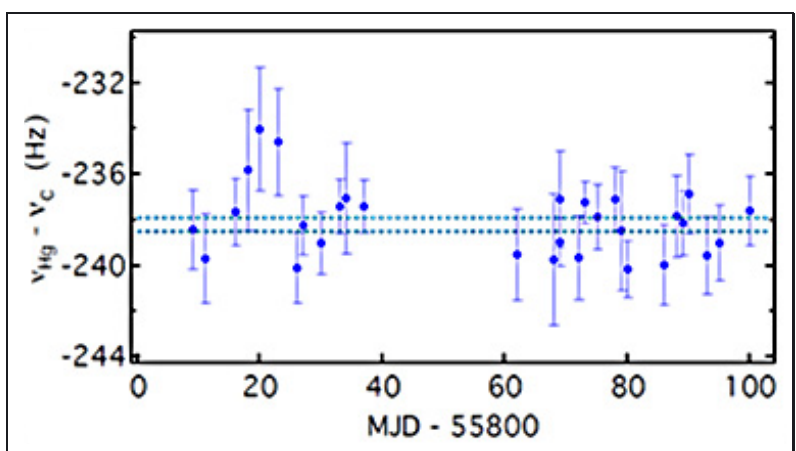

Fig. 24. - Mesures absolues de la fréquence de l'horloge à réseau optique ${ }^{199} \mathrm{Hg}$ par rapport aux références primaires du LNE-SYRTE. Les mesures ont été effectuées sur une durée d'environ 3 mois; MJD : Jour julien modifié; $v_{C}=$ $1128575290808400 \mathrm{~Hz}$.

recommandées des fréquences étalons et a été adoptée par le CIPM en juin 2013. La valeur recommandée est $1128575290808162 \mathrm{~Hz}$ avec une incertitude relative recommandée de $1,7 \times 10^{-14}$ égale à 3 fois l'incertitude de notre détermination, conformément aux règles du Groupe de travail commun au Comité Consultatif des Longueurs (CCL) et au CCTF sur les étalons de fréquence. Pour plus d'informations, on peut se reporter au site Internet du Bureau International des Poids et Mesures www.bipm.org/ en/publications/mep.html.

\section{Conclusions}

Nous avons décrit la première phase du développement d'une horloge à réseau optique à atomes de mercure. Au démarrage de ce développement, le refroidissement laser et la spectroscopie laser de la transition horloge n'avait jamais été faits. Depuis, nous avons réalisé une horloge à réseau optique à atomes de mercure, démontrant ainsi sa faisabilité. Nous avons réalisé des mesures absolues de la fréquence de l'horloge, ce qui a conduit à inclure la transition ${ }^{1} \mathrm{~S}_{0}{ }^{-}{ }^{3} \mathrm{P}_{0}$ de ${ }^{199} \mathrm{Hg}$ dans la liste des valeurs recommandées des fréquences étalons adoptée par le CIPM.

Après cette première phase et compte tenu des nombreux progrès faits dans la compréhension de la physique des horloges à réseau optique, on constate que les motivations du choix de l'atome de mercure restent valables et même confortées. Les propriétés du mercure sont favorables pour réduire significativement les principales sources d'incertitude des meilleures horloges optiques du moment. Il y a maintenant la perspective de réaliser une horloge reproductible à mieux que $10^{-18}$ sans cryogénie et avec une enceinte à vide simple et de petite taille, voire avec l'enceinte existante. Obtenir une telle incertitude requerra une mesure précise de la sensibilité au rayonnement thermique et un environnement thermique mieux contrôlé, mais avec une exigence toujours nettement moindre que pour $\mathrm{Sr}$ et $\mathrm{Yb}$, pour la même incertitude.

Un des défis pour la réalisation d'une horloge avec des atomes de mercure est lié aux performances des 
sources laser ultraviolettes. Et de fait, l'avancée des travaux que l'on a décrits a été principalement et fortement limitée par la précarité du fonctionnement de la source laser de refroidissement à $253,7 \mathrm{~nm}$. Mais l'arrivée récente d'une nouvelle technologie de laser à fibre, beaucoup plus fiable, beaucoup plus compacte et même plus puissante, va permettre de remplacer le laser à disque, principal point faible du système. Avec cette nouvelle source, la phase suivante du projet visera à atteindre rapidement l'incertitude limite accessible sans mesure précise de la sensibilité au rayonnement thermique et à réaliser une mesure absolue à la limite d'exactitude des fontaines atomiques. Cela permettra à la transition ${ }^{1} \mathrm{~S}_{0}{ }^{3} \mathrm{P}_{0}$ de ${ }^{199} \mathrm{Hg}$ d'acquérir le statut de «Représentation Secondaire de la Seconde ». Ensuite, on cherchera à obtenir les incertitudes ultimes que permettent les propriétés favorables du mercure. Cela permettra d'utiliser l'horloge à réseau optique de mercure pour tester les lois fondamentales de la physique, pour explorer les nouvelles applications des horloges à la géodésie chronométrique et aux sciences de la Terre, et pour contribuer aux travaux vers une possible redéfinition de la seconde du Système international d'unités. L'horloge sera également utilisée dans le cadre de la mission spatiale PHARAO/ACES et du déploiement des liens optiques fibrés dédiés aux comparaisons de fréquence et de temps.

\section{Remerciements}

Les travaux que l'on a décrits ont été réalisés au LNE-SYRTE, Observatoire de Paris, PSL Research University, CNRS, Sorbonne Universités, UPMC Univ. Paris 06. En plus du soutien et du financement de ces tutelles, Ces travaux ont bénéficiés de financements du CNES, de l'IFRAF/nanoK, de la Ville de Paris, de l'ERC-AdOC, du JRP ITOC de l'EMRP/EMPIR. Ils ont bénéficié de la visite d'un scientifique (D Magalhães) de l'Université de São Paulo/São Carlos, Brésil. Les travaux ont bénéficiés de nombreuses contributions des services informatique, électronique, mécanique et administratif du SYRTE. Le laboratoire SYRTE, SYstèmes de Référence Temps-Espace, est UMR 8630 Il est membre du Labex FIRST-TF, de l'IFRAF/nanoK soutenu par la Région Ile de France. Le LNE, Laboratoire national de métrologie et d'essais, est l'institut national de métrologie français.

\section{Références}

[1] HaLl J.L., "Nobel lecture: Defining and measuring optical frequencies", Rev. Mod. Phys., 78, 2006, 1279.

[2] HÄNSCH T.W., "Nobel lecture: Passion for precision". Rev. Mod. Phys., 78, 2006, 1297.

[3] LE CoQ Y., et al., "Peignes de fréquences femtosecondes pour la mesure des fréquences optiques". Revue Française de Métrologie, 32, 2012, 35.

[4] DIDDAMS S.A., "The evolving optical frequency comb [invited]", J. Opt. Soc. Am. B, 27, 2010, B51.

[5] CHU S., "Nobel lecture: The manipulation of neutral particles". Rev. Mod. Phys., 70, 1998, 685.

[6] Cohen-TannoudjI C.N., "Nobel lecture: Manipulating atoms with photons". Rev. Mod. Phys., 70, 1998, 707.

[7] PHILLIPS W.D., "Nobel lecture: Laser cooling and trapping of neutral atoms”. Rev. Mod. Phys., 70, 1998, 721.
[8] Cornell E.A. et WiEman C.E., "Nobel lecture: BoseEinstein condensation in a dilute gas, the first 70 years and some recent experiments". Rev. Mod. Phys., 74, 2002, 875.

[9] KeTtERLE W., "Nobel lecture: When atoms behave as waves: Bose-Einstein condensation and the atom laser". Rev. Mod. Phys., 74, 2002, 1131.

[10] Clairon A., Dahmani B. et Rutman J., "Accurate absolute frequency measurements on stabilized $\mathrm{CO}_{2}$ and $\mathrm{He}$ Ne infrared lasers", IEEE Trans. Instrum. Meas., 29, 1980, 268.

[11] Clairon A., Dahmani B., Filimon A. et Rutman J., "Precise frequency measurements of $\mathrm{CO}_{2} / \mathrm{OsO}_{4}$ and $\mathrm{HeNe} / \mathrm{CH}_{4}$-stabilized lasers", IEEE Trans. Instrum. Meas., 34, 1985, 265.

[12] Clairon A., Dahmani B., Acef O., Granveaud M., DOMNIN Y.S., POUChKINE S.B., TATARENKOV V.M., et FELDER R., "Recent experiments leading to the characterization of the performance of portable $(\mathrm{He}-\mathrm{Ne}) / \mathrm{CH}_{4}$ lasers: Part ii: Results of the 1986 LPTF absolute frequency measurements", Metrologia, 25, 1988, 9.

[13] Courtillot I., Quessada A., Kovacich R., Brusch A., KolKer D., ZONDY J.-J., ROVERA G., et LEMONDE P., "Clock transition for a future optical frequency standard with trapped atoms", Phys. Rev. A, 68, 2003, 030501.

[14] Brusch A., Le Targat R., Baillard X., Fouché M. et LEMONDE P., "Hyperpolarizability effects in a $\mathrm{Sr}$ optical lattice clock", Phys. Rev. Lett., 96, 2006, 103003.

[15] WestergaARd P.G., LODEWyCK J., LORINI L., LeCAllier A., Burt E.A., Zawada M., Millo J. et LEMONDE P., "Lattice-induced frequency shifts in $\mathrm{Sr}$ optical lattice clocks at the $10^{-17}$ level", Phys. Rev. Lett., 106, 2011, 210801.

[16] Le Targat R., Lorini L., Le CoQ Y., Zawada M., GuÉNA J., AbGRall M., GuRov M., Rosenbusch P., ROVERA D.G., NAGÓRnY B., GARTMAN R., WestergaARD P.G., TOBAR M.E., LOURS M., Santarelli G., Clairon A., Bize S., Laurent P., LEMONDE P. et LODEWYCK J., "Experimental realization of an optical second with strontium lattice clocks", Nat. Commun., 4, 2013, 2109.

[17] LODEWYCK J., ZAWADA M., LORINI L., GUROV M., et LEMONDE P., "Observation and cancellation of a perturbing dc Stark shift in strontium optical lattice clocks", IEEE Trans. Ultrason. Ferroelect. Freq. Contr., 59, 2012, 411.

[18] Middelmann T., Falke S., Lisdat C. et SterR U., "High accuracy correction of blackbody radiation shift in an optical lattice clock", Phys. Rev. Lett., 109, 2012, 263004.

[19] Sherman J.A., Lemke N.D., Hinkley N., Pizzocaro M., Fox R.W., Ludlow A.D. et OATES C.W., "Highaccuracy measurement of atomic polarizability in an optical lattice clock", Phys. Rev. Lett., 108, 2012, 153002.

[20] Angstmann E.J., Dzuba V.A. et Flambaum V.V., "Relativistic effects in two valence-electron atoms and ions and the search for variation of the fine-structure constant", Phys. Rev. A, 70, 2004, 014102. 
[21] MeJri S., McFerran J.J., Yi L., Le CoQ Y. et Bize S., "Ultraviolet laser spectroscopy of neutral mercury in a one-dimensional optical lattice", Phys. Rev. A, 84, 2011, 032507.

[22] McFerran J.J., Yi L., MeJRi S. et Bize S., "Sub-Doppler cooling of fermionic $\mathrm{Hg}$ isotopes in a magneto-optical trap", Opt. Lett., 35, 2010, 3078.

[23] BLACK E.D., "An introduction to Pound-Drever-Hall laser frequency stabilization", Am. J. Phys. 69, 2001, 79.

[24] Dawkins S., Chicireanu R., Petersen M., Millo J., Magalhes D., Mandache C., Le CoQ Y. et Bize S., "An ultra-stable referenced interrogation system in the deep ultraviolet for a mercury optical lattice clock", Appl. Phys. B: Lasers and Optics, 99, 2010, 41.

[25] Millo J., Magalhães D.V., Mandache C., CoQ Y.L., ENGLISH E.M.L., WESTERGAARD P.G., LODEWYCK J., Bize S., LEMONDE P. et SANTARELli G., "Ultrastable lasers based on vibration insensitive cavities", Phys. Rev. A, 79, 2009, 053829.

[26] Numata K., Kemery A. et CAMP, J., "Thermal-noise limit in the frequency stabilization of lasers with rigid cavities", Phys. Rev. Lett., 93, 2004, 250602.

[27] Argence B., Prevost E., LÉvèQue T., Le Goff R., BIZE S., LEMONDE P. et SANTARELli G., "Prototype of an ultra-stable optical cavity for space applications", Opt. Express, 20, 2012, 25409.

[28] Chambon D., Bize S., Lours M., Narbonneau F., Marion H., Clairon A., SANTARElli G., Luiten A. et TOBAR M., "Design and realization of a flywheel oscillator for advanced time and frequency metrology", Rev. Sci. Instrum., 76, 2005, 094704.

[29] Guéna J., AbGrall M., Rovera D., Laurent P., Chupin B., Lours M., Santarelli G., Rosenbusch P., Tobar M., Li R., Gibble K., Clairon A. et BIZE S., "Progress in atomic fountains at LNE-SYRTE", IEEE Transactions on Ultrasonics, Ferroelectrics and Frequency Control, 59, 2012, 391.

[30] BizE S. et al., «Réalisation et diffusion de la seconde au LNE-SYRTE fondés sur l'utilisation d'horloges en fontaine atomique », Revue Française de Métrologie, 18, 2009, 13.
[31] Burns K., AdAMs K.B. et Longwell J., "Interference measurements in the spectra of neon and natural mercury”, J. Opt. Soc. Am., 40, 1950, 339.

[32] BuRnS K. et ADAMS K.B., "Energy levels and wavelengths of the isotopes of mercury -199 and -200 ", $J$. Opt. Soc. Am., 42, 1952, 716.

[33] BURns K. et ADAMS K.B., "Energy levels and wavelengths of the isotopes of mercury -198 and -202 ", $J$. Opt. Soc. Am., 42, 1952, 56.

[34] SAnsonetti C.J., SALIT M.L. et ReAdER J., "Wavelengths of spectral lines in mercury pencil lamps", Appl. Opt., 35, 1996, 74.

[35] Saloman E.B., "Wavelengths, energy level classifications, and energy levels for the spectrum of neutral mercury", J. Phys. Chem. Ref. Data, 35, 2006, 1519.

[36] Petersen M., Chicireanu R., Dawkins S.T., Magalhães D.V., Mandache C., Le Coq Y., Clairon A. et BIZE S., "Doppler-free spectroscopy of the ${ }^{1} \mathrm{~S}_{0}{ }^{3} \mathrm{P}_{0}$ optical clock transition in laser-cooled fermionic isotopes of neutral mercury", Phys. Rev. Lett., 101, 2008, 183004.

[37] Bourzeix S., Plimmer M., Nez F., Julien L. et BIRABEN F., "Efficient frequency doubling of a continuous wave titanium: sapphire laser in an external enhancement cavity", Opt. Commun., 99, 1993, 89-94.

[38] Yi L., MejRi S., McFerran J.J., Le CoQ Y. et Bize S., "Optical lattice trapping of ${ }^{199} \mathrm{Hg}$ and determination of the magic wavelength for the ultraviolet ${ }^{1} \mathrm{~S}_{0_{-}}{ }^{3} \mathrm{P}_{0}$ clock transition", Phys. Rev. Lett., 106, 2011, 073005.

[39] McFerran J.J., Yi L., MeJRi S., Di Manno S., Zhang W., Guena J., LE COQ Y. et BIZE, S., "Neutral atom frequency reference in the deep ultraviolet with a fractional uncertainty $=5.7 \times 10^{-15}$ ", Phys. Rev. Lett., 108, 2012, 183004.

[40] McFerran JJ., Magalhães D.V.M., Mandache C., MiLlo J., ZhaNG W., LE COQ Y., SANTARElli G. et BizE S., "Laser locking to the ${ }^{199} \mathrm{Hg}{ }^{1} \mathrm{~S}_{0}{ }^{3} \mathrm{P}_{0}$ clock transition with $5.4 \times 10^{-15} / \sqrt{ } \tau$ fractional frequency instability", Opt. Lett., 37, 2012, 3477.

[41] Hachisu H., Miyagishi K., Porsev S.G., Derevianko A., OVSIANNIKOV V.D., PAL'CHIKOV V.G., TAKAMOTO M. et KATORI H., "Trapping of neutral mercury atoms and prospects for optical lattice clocks", Phys. Rev. Lett., 100, 2008, 053001

[42] Safronova M., Jiang D., Arora B., Clark C., KOZlOV M., SAFrONOVA U. et JOHNSON W., "Blackbody radiation shifts and theoretical contributions to atomic clock research", IEEE Trans. Ultrason. Ferroelec. Freq. Contr., 57, 2010, 94. 